\title{
MULTIPLICITY AND BOUNDARY BEHAVIOR OF QUASIREGULAR MAPS
}

\author{
ANTTI RASILA
}

University of Helsinki, Department of Mathematics and Statistics

\section{Academic dissertation}

To be presented, with the permission of the Faculty of Science of the University of Helsinki, for public criticism in Auditorium CK112, Exactum, on May 27th, 2005, at 12 o'clock. 



\section{ACKNOWLEDGEMENTS}

I express my sincere gratitude to my teacher Professor Matti Vuorinen for introducing me to this subject, for his inspiring guidance and continuous encouragement. I am grateful to Docent Visa Latvala and Dr. Xiao Zhong for carefully reading my manuscript and giving much valuable advice and many suggestions. I am also grateful to Professor Peter Lappan, Michigan State University, for his contribution to this work which enhances the value of the results presented here.

I am indebted to the Finnish Academy of Science and Letters (Vilho, Yrjö ja Kalle Väisälän rahasto) and to the University of Helsinki for financial support.

I thank my friends and colleagues at the Department of Mathematics for many interesting discussions and for providing an outstanding working atmosphere.

Finally, I express my deepest thanks to my parents Ritva and Urpo and my brother Tuomas for their encouragement and support.

Helsinki, May 2005

Antti Rasila 


\section{Contents}

Acknowledgements 3

1. Introduction 5

2. Lindelöf-type theorems 6

3. Main results $\quad 7$

References $\quad 9$ 


\section{INTRODUCTION}

This dissertation consists of the summary and the article [Ras05] which is to be published in the journal Mathematische Zeitschrift. This work is also available in electronic form on the web page:

$$
\text { http://ethesis.helsinki.fi. }
$$

The original publication is available at www.springerlink.com. In the article we consider quasiregular maps in the $n$-dimensional Euclidean space $\mathbb{R}^{n}$. In particular, we study the relation between the multiplicity and the boundary behavior. Our goal is to prove generalizations for a classical theorem of E. Lindelöf. Multiplicity, roughly speaking, means the cardinality of the preimage of a point.

Quasiconformal and quasiregular maps in $\mathbb{R}^{n}$ are respectively natural generalizations of conformal and analytic functions of one complex variable. Quasiregular homeomorphisms, i.e. quasiconformal maps, are characterized by the property that there exists a constant $C \geq 1$ such that small spheres are mapped onto small ellipsoids with the ratio of the larger semiaxis to the smaller one bounded from above by $C$.

Quasiconformal maps were first introduced in the two-dimensional case by H. Grötzsch in 1928 and in the higher dimensions by M. A. Lavrent'ev in 1938. The systematic study of quasiconformal maps in $\mathbb{R}^{n}$ was begun by F. W. Gehring [Geh61] and J. Väisälä [Väi61] in 1961. Quasiregular maps were first introduced by Russian mathematician Yu. G. Reshetnyak in 1960's (see e.g. [Res89]). His work was further developed by Finnish mathematicians O. Martio, S. Rickman and J. Väisälä (e.g. [MRV69]). Geometric concepts originating from the theory of quasiconformal maps were introduced by them to the theory of quasiregular maps. This new point of view combined with Gehring's and Väisälä's work in the field of quasiconformal maps (see e.g.[Geh62], [Väi71]), provided important tools for the research of quasiregular maps. A comprehensive reference on these results is [Ric93]. Another important reference is [Vuo88], where the focus is on methods involving Möbius transformations and conformal invariants. Results 
from these two books are used through the work. Our study in the theory of quasiregular maps builds on the research traditions in the Helsinki School of Analysis in geometric function theory, complex analysis and quasiconformal maps.

\section{LINDELÖF-TYPE THEOREMS}

The following result is known in the literature as Lindelöf's Theorem (see e.g. [Rud87, p.259]):

2.1. Theorem. Suppose that $\gamma$ is a curve, with parameter interval $[0,1]$, such that $|\gamma(t)|<1$ if $t<1$ and $\gamma(1)=1$. If $f$ is a bounded analytic function of the unit disk and

$$
\lim _{t \rightarrow 1} f(\gamma(t))=\alpha
$$

then $f$ has angular limit $\alpha$ at 1, i.e. limit in each cone contained in the unit disk with the vertex in 1.

There are various generalizations of Lindelöf's theorem in the literature. Another question is if a weaker condition would be sufficient for the result. P. Koebe proved that if a bounded analytic function tends to zero along a sequence of arcs in the unit disk which approach a subarc on the boundary, then it must be identically zero [Bie31, p.19].

The bibliography of [Vuo85] lists several authors who have studied this topic. One of them is D. C. Rung, who studied the connection between the boundary behavior and the hyperbolic metric in [Run68]. In Rung's results, the values attained by the function are assumed to approach a limit at a certain rate on a sequence of continua of given hyperbolic diameters. By studying the balance between the rate of convergence and the growth of the hyperbolic diameter, one can make conclusions about the limit behavior of the function.

For example, Rung proved the following result. A path $\gamma:[0,1) \rightarrow D$ is called a boundary path if $|\gamma(t)| \rightarrow 1$ as $t \rightarrow 1$. Let $M(r, f)=\max \left\{\sup _{|z|<r} \log |f(z)|, 1\right\}$.

2.2. Theorem. [Run68, Corollary 1] Suppose that $\gamma$ is a boundary path in the unit disk $D$, and $f$ is analytic in $D$ such that for some finite $w_{0}$ and some positive function $A(r), r \in[0,1)$,

$$
\left|f(z)-w_{0}\right| \leq \exp \left(\frac{-A(r)}{(1-|z|)}\right),
$$

for $z \in|\gamma|,|z| \geq r$ and

$$
\liminf _{r \rightarrow 1} M(r, f) / A(r)=0,
$$

then $f \equiv w_{0}$. 
2.3. Remark. We may choose a sequence $\left\{\gamma_{k}\right\}$ of subpaths of $\gamma$ such that

$$
\begin{aligned}
1 / 2 & \leq \min _{z \in\left|\gamma_{k}\right|}|z|=r_{k} \rightarrow 1 \text { as } k \rightarrow \infty, \\
0 & <\liminf _{k \rightarrow \infty} \rho\left(\left|\gamma_{k}\right|\right) \leq \limsup _{k \rightarrow \infty} \rho\left(\left|\gamma_{k}\right|\right)<\infty,
\end{aligned}
$$

where $\rho\left(\left|\gamma_{k}\right|\right)$ is the hyperbolic diameter of $\left|\gamma_{k}\right|$. The sets $\left|\gamma_{k}\right|$ take the role of the Koebe arcs in this result. The function $A(r)$ gives the required speed of convergence.

A very recent survey of the results of this type in the plane is given in [EE02].

Since the quasiconformal maps and quasiregular maps are natural generalizations of conformal and analytic maps, respectively, the question arises as to whether Lindelöf's theorem holds for them as well. For the quasiconformal maps, Gehring's result (see [Geh63, p.21]) shows that this is indeed the case.

The situation is different for non-homeomorphic quasiregular mappings. The counter-example of Rickman [Ric80] (see also [Ric93, p.193]) gives a bounded quasiregular mapping $f$ of the unit ball $\mathbf{B}^{n}, n \geq 3$ and a point $b$ such that

(1) $f$ has infinitely many asymptotic values at $b$,

(2) $f$ has no angular limit at $b$.

Against this background, the question is: what results, if any, of this type can be proved for quasiregular maps?

This question about the existence of angular limits has been studied by M. Vuorinen in a series of articles in the 1970's and 1980's (see [Vuo81], [Vuo82] and [Vuo85]). A survey article summarizing the results known in 1983 is [Vuo83]. An article of particular importance is [Vuo85], where Vuorinen outlines a research plan that could be carried out provided that the following open problem could be solved.

2.4. Question. [Vuo85, 3.8], [Vuo88, p. 193] Let $n \geq 2, K \geq 1$, and $\delta \in(0,1)$. Does there exist a number $d_{0}=d_{0}(n, K, \delta)$ such that if $f: \mathbf{B}^{n} \rightarrow \mathbf{B}^{n}$ is a nonconstant $K$-quasiregular mapping with $|f(0)| \geq \delta$, then card $Z \leq d_{0}$, where $Z=\left\{x \in \mathbf{B}^{n}:|x| \leq 1 / 2, f(x)=0\right\} ?$

This question was recently affirmatively answered by P. Järvi [Jär00]. Järvi proved for quasiregular maps a variant of the Schwarz lemma with multiplicity. The remarkable results of Järvi put the paper [Vuo85] into a new light and were the main motivation for this study. His work along with the methods developed by Rickman and Vuorinen are crucial for this work.

\section{MAin RESUlts}

The main results in this work give criteria for a quasiregular mapping to have a limit along tangential and non-tangential sets.

The next result is closely related to [Vuo85, Theorem 3.6.]. The difference is that the estimates here involve the local topological index $i(x, f)$. This result 
gives a sufficient condition for a quasiregular mapping to have an angular limit and also a stronger condition under which the mapping must be a constant.

3.1. Theorem. [Ras05, 5.4] Let $f: \mathbf{H}^{n} \rightarrow \mathbf{B}^{n}$ be a $K$-quasiregular mapping, let $t_{k}=2^{-k}$ and $f\left(t_{k} e_{n}\right)=0$ for all $k=0,1, \ldots$

(1) If $\limsup _{k \rightarrow \infty} t_{k}^{\gamma} \mu\left(t_{k} e_{n}, f\right)=\infty$, where $\mu\left(t_{k} e_{n}, f\right)=d_{3} i\left(t_{k} e_{n}, f\right)^{1 /(n-1)}$ and $\gamma=d_{6} \log (1 / \beta)$, then $f \equiv 0$.

(2) If $\mu\left(t_{k} e_{n}, f\right) \rightarrow \infty$ as $k \rightarrow \infty$, then $f$ has an angular limit 0 at the origin.

Here $d_{6}>0, \beta \in(0,1)$ and $d_{3} \in(0,1)$ depend only on $n$ and $K$.

We also prove a similar result concerning the existence of a limit in a tangential set. Note that the criterion of this result is between the conditions (1) and (2) of Theorem 3.1 for $\alpha \in(1,1+\log (2) / 2)$.

3.2. Theorem. [Ras05, 5.9] Let $f: \mathbf{H}^{n} \rightarrow \mathbf{B}^{n}$ be a $K$-quasiregular mapping with $f\left(2^{-k} e_{n}\right)=0$, for all $k=1,2, \ldots$ and let $\gamma=d_{6} \log (1 / \beta), \mu_{k}=d_{3} i\left(2^{-k} e_{n}, f\right)^{1 /(n-1)}$, where $\beta \in(0,1), d_{6}>0$ and $d_{3} \in(0,1)$ depend only on $n, K$.

Fix $\alpha>1$. Suppose that

$$
\log \mu_{k}-2 \gamma(\alpha-1) k \rightarrow \infty \text { as } k \rightarrow \infty .
$$

Then $f$ has limit 0 at the origin along the tangential set $E=\left\{x=\left(x_{1}, 0, \ldots, 0, x_{n}\right): x_{n}=x_{1}^{\alpha}\right\}$.

The next result shows an explicit bound for the modulus of continuity of a quasiregular map on a cone contained in the upper half space with the vertex at the origin.

3.3. Theorem. [Ras05, 5.11] Let $f: \mathbf{H}^{n} \rightarrow \mathbf{B}^{n}$ be $K$-quasiregular, $f\left(2^{-j} e_{n}\right) \rightarrow$ $\alpha$, with $i\left(2^{-j} e_{n}, f\right)^{1 /(n-1)}=j$, and let $x \in C(\varphi)$ for some $\varphi \in(0, \pi / 2)$. Let $k$ be such that $2^{-k-1}<|x| \leq 2^{-k}$. Then

$$
|f(x)-\alpha| \leq M(|x|, n, K, \varphi) \rightarrow 0 \text { as }|x| \rightarrow 0,
$$

where

$$
M(|x|, n, K, \varphi)=2\left(\frac{2-s}{1-s}\right) s^{k} \leq \frac{4}{s(1-s)}|x|^{-\log _{2} s},
$$

$R_{\varphi}>0$ is a constant depending only on $\varphi, s=(2 / 3)^{d_{3} A} \in(0,1), A=\beta^{1+d_{6} R_{\varphi}} \in$ $(0,1)$ depend only on $n, K, \varphi$ and $\beta \in(0,1), d_{3} \in(0,1), d_{6}>1$ are constants depending only on $n, K$. In particular, $-\log _{2} s=d_{3} A \log _{2}(3 / 2) \in(0,1)$.

In addition, we prove the following result, where a criterion for a quasiregular map to be constant is given in terms of the local topological index on a sequence of points taken from a cone. 
3.4. Theorem. [Ras05, 5.15] Let $f: \mathbf{H}^{n} \rightarrow \mathbf{B}^{n}$ be $K$-quasiregular, $\varphi \in(0, \pi / 2)$, and let $\left(b_{k}\right), f\left(b_{k}\right)=0$ be a sequence of points in $\mathbf{H}^{n}$ with $b_{k} \rightarrow 0$ as $k \rightarrow \infty$. If

$$
i\left(b_{k}, f\right)\left(b_{k}\right)_{n}^{d_{10}} \rightarrow \infty
$$

as $k \rightarrow \infty$, then $f \equiv 0$. Here $d_{10}>0$ is a constant depending only on $n, K$.

In particular, if the points $\left(b_{k}\right)$ are in $C(\varphi)$ and

$$
i\left(b_{k}, f\right)\left|b_{k}\right|^{d_{10}} \rightarrow \infty
$$

as $k \rightarrow \infty$, then $f \equiv 0$.

We also prove an improved version of [Vuo85, Corollary 4.9], which deals with quasiconformal maps. The improvement is due to the improved estimate for the modulus obtained by using the Teichmüller ring modulus function $\tau(s)$.

3.5. Theorem. $[\operatorname{Ras} 05,5.34]$ Let $f: \mathbf{B}^{n} \rightarrow \mathbb{R}^{n}$ be a quasiconformal mapping or constant, $\alpha \in \mathbb{R}^{n}$ and $\left(C_{j}\right)$ a sequence of nondegenerate continua such that $C_{j} \rightarrow \partial \mathbf{B}^{n}$ and $|f(x)-\alpha|<M_{j}$ when $x \in C_{j}$, where $M_{j} \rightarrow 0$ as $j \rightarrow \infty$. If

$$
\limsup _{j \rightarrow \infty} \tau\left(\frac{1}{d\left(C_{j}\right)}\right)\left(\log \frac{1}{M_{j}}\right)^{n-1}=\infty,
$$

then $f \equiv \alpha$. In particular, if

$$
\limsup _{j \rightarrow \infty}\left(\log \frac{1}{d\left(C_{j}\right)}\right)^{1-n}\left(\log \frac{1}{M_{j}}\right)^{n-1}=\infty
$$

then $f \equiv \alpha$.

\section{REFERENCES}

[Bie31] L. BIEBERBACH: Lehrbuch der Funktionentheorie, Bd. 2, Moderne Funktionentheorie, Leipzig, Teubner, 1931.

[EE02] V. EIDERMAN AND M. EssÉn: Uniqueness Theorems of Analytic and Subharmonic Functions, Algebra i Analiz 14, no. 6 (2002), 1-88.

[Geh61] F. W. GeHRING: Symmetrization of rings in space, Trans. Amer. Math. Soc. 101 (1961) 499-519.

[Geh62] F. W. GeHrIng: Rings and quasiconformal mappings in space, Trans. Amer. Math. Soc. 103 (1962) 137-150.

[Geh63] F. W. GeHRING: The Carathéodory convergence theorem for quasiconformal mappings in the space, Ann. Acad. Sci. Fenn. Ser. A I 336/11 (1963), 1-21.

[Jär00] P. J̈̈RVI: On the zeros and growth of quasiregular mappings, J. Anal. Math. 82 (2000), 347-362.

[MRV69] O. Martio, S. RiCkman And J. Väıs̈̈L̈̈: Definitions for quasiregular mappings, Ann. Acad. Sci. Fenn. Ser. A I Math. 448 (1969), 1-40.

[Ras05] A. RAsila: Multiplicity and boundary behavior of quasiregular maps, to be published in Math. Z., 2005.

[Res89] Yu. G. Reshetnyak: Space Mappings with Bounded Distortion, Translations of Mathematical Monographs 73, Amer. Math. Soc., Providence, 1989. 
[Ric80] S. Rickman: Asymptotic values and angular limits of quasiregular mappings of a ball, Ann. Acad. Sci. Fenn. Ser. A I Math. 5 (1980), 185-196.

[Ric93] S. Rickman: Quasiregular Mappings, Ergeb. Math. Grenzgeb. (3), Vol. 26, Springer-Verlag, Berlin, 1993.

[Rud87] W. Rudin: Real and complex analysis, New York, McGraw-Hill, 1987.

[Run68] D. C. RunG: Behavior of holomorphic functions in the unit disk on arcs of positive diameter, J. Math. Kyoto Univ. 8-3 (1968), 417-464.

[Väi61] J. VÄIsÄLÄ: On quasiconformal mappings in space, Ann. Acad. Sci. Fenn. Ser. A I 298 (1961), 1-36.

[Väi71] J. VÄIsÄLÄ: Lectures on n-Dimensional Quasiconformal Mappings, Lecture Notes in Math., Vol. 229, Springer-Verlag, Berlin, 1971.

[Vuo81] M. Vuorinen: Capacity densities and angular limits of quasiregular mappings, Trans. Amer. Math. Soc. 263 (1981), 343-354.

[Vuo82] M. VuORINEn: On the Harnack constant and the boundary behavior of Harnack functions, Ann. Acad. Sci. Fenn. Ser. A I Math. 7 (1982), 259-277.

[Vuo83] M. VUORINEN: Lindelöf-type theorems for quasiconformal and quasiregular mappings, Complex analysis (Warsaw, 1979), 353-362, Banach Center Publ., 11, PWN, Warsaw, 1983.

[Vuo85] M. Vuorinen: Koebe Arcs and Quasiregular Mappings, Math. Z. 190 (1985), 95106.

[Vuo88] M. Vuorinen: Conformal Geometry and Quasiregular Mappings, Lecture Notes in Math., Vol. 1319, Springer-Verlag, Berlin, 1988. 


\title{
MULTIPLICITY AND BOUNDARY BEHAVIOR OF QUASIREGULAR MAPS
}

\author{
ANTTI RASILA
}

To appear in Mathematische Zeitschrift

\author{
The original publication is available at \\ www.springerlink.com.
}





\title{
MULTIPLICITY AND BOUNDARY BEHAVIOR OF QUASIREGULAR MAPS
}

\author{
ANTTI RASILA
}

\begin{abstract}
We study the boundary behavior of a bounded quasiregular mapping $f: G \rightarrow \mathbb{R}^{n}$. In the main results, Lindelöf-type problems are studied in connection with the local topological index $i(x, f)$. The existence of certain types of limits at a given boundary point $b \in \partial G$ is shown. The assumptions involve local topological index of the mapping $f$ on a given sequence of points approaching the boundary point $b$.
\end{abstract}

\section{INTRODUCTION}

The subject of this paper is the theory of quasiregular mappings in the $n$ dimensional Euclidean space $\mathbb{R}^{n}$. Quasiregular mappings were first introduced by Yu. G. Reshetnyak in a series of papers in the 1960's. The theory was further developed by a group of Finnish mathematicians, O. Martio, S. Rickman and J. Väisälä. Quasiregular mappings are a natural generalization of complex analytic functions in the geometric sense. The theory extends the theory of analytic functions in the plane and also generalizes to $\mathbb{R}^{n}$. In fact, many of the important results concerning quasiregular mappings are counterparts of the classical theorems of complex analysis. Furthermore, this new point of view contributes to the deeper understanding of the classical theory as well. Some of the main results of the theory of quasiregular maps can be found in the monographs [Res89], [Ric93] and [Vuo88].

A mapping is said to have an angular limit at a boundary point, if it has a limit in each cone with vertex at this point. A function has an asymptotic value at a boundary point $b$ if it has a limit along a curve terminating at $b$. It is a classical result by E. Lindelöf that a conformal mapping of the unit disk, having an asymptotic value $\alpha$ at a boundary point $b$, also has an angular limit $\alpha$ at $b$. Generalizations of the Lindelöf theorem can be found in the literature, e.g. a similar result for quasiconformal mappings in $\mathbb{R}^{3}$ was proved by F. W. Gehring in [Geh63]. Because Lindelöf's theorem also holds for bounded analytic functions, it was a natural question to explore whether a similar result holds for bounded quasiregular maps of the unit ball in $\mathbb{R}^{n}$ for $n \geq 3$. Rickman solved this question by proving [Ric80b, Theorem 1.1] that this is not the case for $n \geq 3$.

P. Koebe proved that if a bounded analytic function $f$ tends to zero along a sequence of arcs in the unit disk which approach a subarc in the boundary, then $f$ must be identically zero [Bie31, p.19]. Refinements of Koebe's results 
were given by D. C. Rung in [Run68], where the relation between the hyperbolic metric and the boundary behavior of an analytic function was studied. In Rung's results, the values attained by the function are assumed to approach a limit at a certain rate on a sequence of continua of given hyperbolic diameter. By studying the balance between the rate of convergence and the growth of the hyperbolic diameter, one can make conclusions on the limit behavior of the function. An extensive survey of Koebe-type results was recently published by V. Eiderman and M. Essén [EE02].

M. Vuorinen [Vuo85], motivated by the work of Koebe, Rung and others, proved similar results for quasiregular maps in $\mathbb{R}^{n}$. In [Vuo85] Vuorinen also raised an important question about the relationship between the growth and the multiplicity of a bounded quasiregular mapping [Vuo85, p.100]. Very recently, P. Järvi [Jär00] found an elegant solution to this question. Järvi's work posed the paper [Vuo85] in a new perspective. In this paper, we apply Järvi's work in [Jär00] to refine the results in [Vuo85]. For the purpose of easy reference, this problem is given below in 3.1 and its solution in Corollary 3.7.

In what follows our main method is to combine Järvi's result with Rickman's two-constants theorem. By applying this technique, we obtain improvements and new variants of the various results for quasiregular mappings in $\mathbb{R}^{n}$.

This paper is organized as follows:

In Section 2 we briefly introduce the basic tools, notation and terminology used through this paper. The concepts introduced there include hyperbolic metrics, local topological index, conformal modulus and capacity. Capacity inequalities are the most important tools in the study of quasiregular mappings. Capacity and radial densities are also introduced in Section 2. These quantities are used in measuring the thickness of a set at a point in the sense of the conformal capacity.

The main topic in Section 3 is the influence of the local topological index on the value distribution of quasiregular mappings. This problem is studied in the light of Järvi's recent results. Järvi's version of Schwarz lemma for quasiregular mappings is presented here, together with some applications. For instance, we prove a result of the same type as the main theorem of [RS86].

In Section 4, we apply Rickman's two-constants theorem [Ric80a, Theorem 4.22] and combine it with Järvi's results from Section 3. By means of the twoconstants theorem we extend Järvi's local Schwarz lemma to a global version. As applications, a few lemmas related to the measure density are also proved in this section. Measure densities are quantities related to the measure theoretic thickness of a set at a given point. They are related to the capacity and radial densities introduced in Section 2.

The main results are presented in Section 5. Here the methods from Section 4 are applied to study the connection between the local topological index and the limit behavior of the function. The study of the limit behavior involves several different concepts of a limit. In the main results, a sequence of points 
approaching the boundary is studied. The function is assumed to have a limit along the given sequence, and the topological indices are required to grow to infinity at a sufficient rate. Some of our results are sharp as shown by an example of P. Lappan [Lap03].

We obtain sufficient conditions for three types of conclusions:

(1) The function has an angular limit at the boundary point.

(2) The function has a limit along a tangential set at the point.

(3) The function is constant.

This paper was written under supervision of M. Vuorinen.

\section{Notation AND PRELIMINARY RESUlts}

We shall follow standard notation and terminology adopted from [Väi71], [Vuo88] and [Ric93]. For $x \in \mathbb{R}^{n}, n \geq 2$, and $r>0$ let $\mathbf{B}^{n}(x, r)=\left\{z \in \mathbb{R}^{n}\right.$ : $|z-x|<r\}, S^{n-1}(x, r)=\partial \mathbf{B}^{n}(x, r), \mathbf{B}^{n}(r)=\mathbf{B}^{n}(0, r), S^{n-1}(r)=\partial \mathbf{B}^{n}(r)$, $\mathbf{B}^{n}=\mathbf{B}^{n}(1), S^{n-1}=\partial \mathbf{B}^{n}, \mathbf{H}^{n}=\left\{x \in \mathbb{R}^{n}: x_{n}>0\right\}$ and $\mathbf{B}_{+}^{n}=\mathbf{B}^{n} \cap \mathbf{H}^{n}$. The space $\overline{\mathbb{R}}^{n}=\mathbb{R}^{n} \cup\{\infty\}$ is the one-point compactification of $\mathbb{R}^{n}$. The surface area of $S^{n-1}$ is denoted by $\omega_{n-1}$ and $\Omega_{n}$ is the volume of $\mathbf{B}^{n}$. It is well-known that $\omega_{n-1}=n \Omega_{n}$ and that

$$
\Omega_{n}=\frac{\pi^{n / 2}}{\Gamma(1+n / 2)}
$$

for $n=2,3, \ldots$, where $\Gamma$ is Euler's gamma function. The standard coordinate unit vectors are denoted by $e_{1}, \ldots, e_{n}$. The Lebesgue measure on $\mathbb{R}^{n}$ is denoted by $m$.

For nonempty subsets $A$ and $B$ of $\overline{\mathbb{R}}^{n}$, we let $d(A)=\sup \{|x-y|: x, y \in A\}$ be the diameter of $A, d(A, B)=\inf \{|x-y|: x \in A, y \in B\}$ the distance between the sets $A$ and $B$, and we abbreviate $d(\{x\}, B)$ as $d(x, B)$.

2.1. Hyperbolic metrics. The hyperbolic metrics $\rho_{\mathbf{H}^{n}}$ and $\rho_{\mathbf{B}^{n}}$ in the upper half space $\mathbf{H}^{n}$ and the unit ball $\mathbf{B}^{n}$ are defined by

$$
\cosh \rho_{\mathbf{H}^{n}}(x, y)=1+\frac{|x-y|^{2}}{2 x_{n} y_{n}}, x, y \in \mathbf{H}^{n},
$$

and

$$
\sinh ^{2}\left(\frac{1}{2} \rho_{\mathbf{B}^{n}}(x, y)\right)=\frac{|x-y|^{2}}{\left(1-|x|^{2}\right)\left(1-|y|^{2}\right)}, x, y \in \mathbf{B}^{n},
$$

respectively. If there is no danger of confusion, we denote both $\rho_{\mathbf{H}^{n}}(x, y)$ and $\rho_{\mathbf{B}^{n}}(x, y)$ simply by $\rho(x, y)$. A hyperbolic ball with the center $x$ and the radius $M>0$ is denoted by $D(x, M)$.

A well-known fact is that hyperbolic balls are balls in the Euclidean geometry as well. This fact along with the observation that for, $\lambda=e^{M}$, the points $\lambda t e_{n}$, 
$(t / \lambda) e_{n}$ are in the boundary of $D\left(t e_{n}, M\right)$, gives us the following formulas for hyperbolic balls in $\mathbf{H}^{n}$ [Vuo88, (2.11)]:

$$
\left\{\begin{array}{l}
D\left(t e_{n}, M\right)=\mathbf{B}^{n}\left((t \cosh M) e_{n}, t \sinh M\right), \\
\mathbf{B}^{n}\left(t e_{n}, r t\right) \subset D\left(t e_{n}, M\right) \subset \mathbf{B}^{n}\left(t e_{n}, R t\right),
\end{array}\right.
$$

where $r=1-e^{-M}$ and $R=e^{M}-1$.

Both for $\left(\mathbf{B}^{n}, \rho_{\mathbf{B}^{n}}\right)$ and $\left(\mathbf{H}^{n}, \rho_{\mathbf{H}^{n}}\right)$ one can define the hyperbolic distance in terms of the absolute ratio. Since the absolute ratio is invariant under Möbius transformations the hyperbolic metric also remains invariant under these transformations.

2.5. Paths in $\mathbb{R}^{n}$. A path in $\mathbb{R}^{n}$ is a continuous mapping $\alpha: \Delta \rightarrow \mathbb{R}^{n}$, where $\Delta$ is a (possibly unbounded) interval in $\mathbb{R}$. The path $\alpha$ is called closed (open) if $\Delta$ is compact (open). The locus $|\alpha|$ of $\alpha$ is the image set $\alpha \Delta$.

Let $\gamma:[a, b] \rightarrow \mathbb{R}^{n}$ be a closed path. The length $\ell(\gamma)$ of the path $\gamma$ is defined by means of polygonal approximation (see [Väi71], pages 1-8). The path $\gamma$ is called rectifiable if $\ell(\gamma)<\infty$ and locally rectifiable if each closed subpath of $\gamma$ is rectifiable. If $\gamma$ is a rectifiable path, then $\gamma$ has a parameterization by means of arc length, also called the normal representation of $\gamma$. The normal representation of $\gamma$ is denoted by $\gamma^{0}:[0, \ell(\gamma)] \rightarrow \mathbb{R}^{n}$. By making use of the normal representation, one may define the integral over a locally rectifiable path $\gamma$.

2.6. Modulus of a path family. Let $\Gamma$ be a path family in $\mathbb{R}^{n}, n \geq 2$. Let $\mathcal{F}(\Gamma)$ be the set of all Borel functions $\rho: \mathbb{R}^{n} \rightarrow[0, \infty]$ such that

$$
\int_{\gamma} \rho d s \geq 1
$$

for every locally rectifiable path $\gamma \in \Gamma$. The functions in $\mathcal{F}(\Gamma)$ are called admissible for $\Gamma$. For $1 \leq p \leq \infty$ we define

$$
\mathrm{M}_{p}(\Gamma)=\inf _{\rho \in \mathcal{F}(\Gamma)} \int_{\mathbb{R}^{n}} \rho^{p} d m
$$

and call $\mathrm{M}_{p}(\Gamma)$ the $p$-modulus of $\Gamma$. If $\mathcal{F}(\Gamma)=\emptyset$, which is true only if $\Gamma$ contains constant paths, we set $\mathrm{M}_{p}(\Gamma)=\infty$. The $n$-modulus or conformal modulus is denoted by $\mathrm{M}(\Gamma)$.

Let $\Gamma_{1}$ and $\Gamma_{2}$ be path families in $\mathbb{R}^{n}$. We say that $\Gamma_{2}$ is minorized by $\Gamma_{1}$ and write $\Gamma_{1}<\Gamma_{2}$ if every $\gamma \in \Gamma_{2}$ has a subpath in $\Gamma_{1}$. If $\Gamma_{1}<\Gamma_{2}, \mathcal{F}\left(\Gamma_{1}\right) \subset \mathcal{F}\left(\Gamma_{2}\right)$ and hence $\mathrm{M}_{p}\left(\Gamma_{1}\right) \geq \mathrm{M}_{p}\left(\Gamma_{2}\right)$. If $\Gamma_{1} \supset \Gamma_{2}$ then $\Gamma_{1}<\Gamma_{2}$.

The path families $\Gamma_{1}, \Gamma_{2}, \ldots$ are called separate if there exist disjoint Borel sets $E_{i}$ such that

$$
\int_{\gamma} \chi_{\mathbb{R}^{n} \backslash E_{i}} d s=0
$$

for all locally rectifiable $\gamma \in \Gamma_{i}, i=1,2, \ldots$ 
2.9. Lemma. [Ric93, Proposition II.1.5] Let $\Gamma, \Gamma_{1}, \Gamma_{2}, \ldots$ be a sequence of path families in $\mathbb{R}^{n}$. Then

(1) $\mathrm{M}_{p}\left(\bigcup_{i} \Gamma_{i}\right) \leq \sum_{i} \mathrm{M}_{p}\left(\Gamma_{i}\right)$.

(2) If $\Gamma_{1}, \Gamma_{2}, \ldots$ are separate and $\Gamma<\Gamma_{i}$ for all $i$, then

$$
\mathrm{M}_{p}(\Gamma) \geq \sum_{i} \mathrm{M}_{p}\left(\Gamma_{i}\right) .
$$

Equality holds if $\Gamma=\bigcup_{i} \Gamma_{i}$.

(3) If $\Gamma_{1}, \Gamma_{2}, \ldots$ are separate and $\Gamma_{i}<\Gamma$ for all $i$, then

$$
\mathrm{M}_{p}(\Gamma)^{1 /(1-p)} \geq \sum_{i} \mathrm{M}_{p}\left(\Gamma_{i}\right)^{1 /(1-p)}, p>1 .
$$

For $E, F, G \subset \mathbb{R}^{n}$ we denote by $\Delta(E, F ; G)$ the family of all nonconstant paths joining $E$ and $F$ in $G$. If $G=\mathbb{R}^{n}$, we may omit $G$ and simply denote $\Delta\left(E, F ; \mathbb{R}^{n}\right)$ by $\Delta(E, F)$. A domain $G$ in $\overline{\mathbb{R}}^{n}$ is called a ring, if $\overline{\mathbb{R}}^{n} \backslash G$ has exactly two components. If the components are $E$ and $F$, we denote the ring by $R(E, F)$.

In general, it is difficult to calculate the modulus of a given path family. The following two lemmas give us an important tool, letting us obtain effective upper and lower bounds for the modulus in many situations.

2.10. Lemma. [Väi71, 7.5.] Let $0<a<b<\infty, A=\mathbf{B}^{n}(b) \backslash \overline{\mathbf{B}}^{n}(a)$ and

$$
\Gamma_{A}=\Delta\left(S^{n-1}(a), S^{n-1}(b) ; A\right) .
$$

Then

$$
\mathrm{M}\left(\Gamma_{A}\right)=\omega_{n-1}\left(\log \frac{b}{a}\right)^{1-n}
$$

2.11. Canonical ring domains. The complementary components of the Grötzsch ring $R_{G, n}(s)$ in $\mathbb{R}^{n}$ are $\overline{\mathbf{B}}^{n}$ and $\left[s e_{1}, \infty\right], s>1$, and those of the Teichmüller ring $R_{T, n}(s)$ are $\left[-e_{1}, 0\right]$ and $\left[s e_{1}, \infty\right], s>0$. We define two special functions $\gamma_{n}(s), s>1$ and $\tau_{n}(s), s>0$ by

$$
\left\{\begin{array}{l}
\gamma_{n}(s)=\mathrm{M}\left(\Delta\left(\overline{\mathbf{B}}^{n},\left[s e_{1}, \infty\right]\right)\right)=\gamma(s) \\
\tau_{n}(s)=\mathrm{M}\left(\Delta\left(\left[-e_{1}, 0\right],\left[s e_{1}, \infty\right]\right)\right)=\tau(s)
\end{array}\right.
$$

respectively. The subscript $n$ is omitted if there is no danger of confusion. We shall refer to these functions as the Grötzsch capacity and the Teichmüller capacity. It is well-known [Vuo88, Lemma 5.53] that for all $s>1$

$$
\gamma_{n}(s)=2^{n-1} \tau_{n}\left(s^{2}-1\right)
$$

and that $\tau_{n}:(0, \infty) \rightarrow(0, \infty)$ is a decreasing homeomorphism.

2.12. Lemma. [AVV97, (8.65)] The following estimate holds for $\tau_{n}(t), t>0$ :

$$
\tau_{n}(t) \geq 2^{1-n} \omega_{n-1}\left(\log \left(\frac{\lambda_{n}}{2}(\sqrt{1+t}+\sqrt{t})\right)\right)^{1-n},
$$


where $\lambda_{n} \geq 2$ is the Grötzsch constant depending only on $n$.

The value of $\lambda_{n}$ is known only for $n=2$, namely $\lambda_{2}=4$. For $n \geq 3$ it is known that $2^{0.76(n-1)} \leq \lambda_{n} \leq 2 e^{n-1}$. For more information on the Grötzsch ring constant $\lambda_{n}$, see [AVV97, p. 169].

2.13. $\mathrm{ACL}^{p}$ mappings. For $j=1, \ldots, n$ let $R_{j}^{n}=\left\{x \in \mathbb{R}^{n}: x_{j}=0\right\}$ and let $T_{j}: \mathbb{R}^{n} \rightarrow R_{j}^{n}$ be the orthogonal projection $T_{j} x=x-x_{j} e_{j}$. Let $G \subset \mathbb{R}^{n}$ be an open set and $u: G \rightarrow \mathbb{R}^{n}$ a continuous function. The function $u$ is called absolutely continuous on lines (ACL), if for every cube $Q$ with $\bar{Q} \subset G$, the set $A_{j} \subset T_{j} G$ of all points such that the function $t \mapsto u\left(z+t e_{j}\right), z+t e_{j} \in Q$, is not absolutely continuous as a function of single variable, satisfies $m_{n-1}\left(A_{j}\right)=0$ for all $j=1, \ldots, n$.

Such a mapping has partial derivatives $D_{i} f(x)$ a.e. in $G$, and they are Borel functions [Väi71, 26.4]. If $p \geq 1$ and the partial derivatives of $f$ are locally $L^{p}$-integrable, $f$ is said to be in $\mathrm{ACL}^{p}$ or in $\mathrm{ACL}^{p}(G)$.

2.14. Capacity of a condenser. A condenser in $\mathbb{R}^{n}$ is a pair $E=(A, C)$, where $A$ is open in $\mathbb{R}^{n}$ and $C$ is a compact subset of $A$. The p-capacity of $E$ is defined by

$$
\operatorname{cap}_{p} E=\inf _{u} \int_{A}|\nabla u|^{p} d m, \quad 1 \leq p<\infty,
$$

where the infimum is taken over all nonnegative functions $u$ in $\operatorname{ACL}^{p}(A)$ with compact support in $A$ and $u \mid C \geq 1$. The $n$-capacity of $E$ is called the conformal capacity of $E$ and denoted by cap $E$.

2.16. Remark. It is well-known [Ric93, Proposition II.10.2] that

$$
\operatorname{cap}(A, C)=\mathrm{M}(\Delta(C, \partial A ; A))
$$

for all condensers $(A, C)$ in $\mathbb{R}^{n}$.

2.18. Sets of zero capacity. A compact set $E$ in $\mathbb{R}^{n}$ is said to be of capacity zero, denoted cap $E=0$, if there exists a bounded set $A$ with $E \subset A$ and $\operatorname{cap}(A, E)=0$. A compact set $E \subset \overline{\mathbb{R}}^{n}, E \neq \overline{\mathbb{R}}^{n}$ is said to be of capacity zero if $E$ can be mapped by a Möbius transformation onto a bounded set of capacity zero. Otherwise $E$ is said to be of positive capacity, and we write cap $E>0$.

2.19. Capacity and radial densities. In what follows we need a condition for the thickness of a set at a point. For this purpose we introduce the following definition.

2.20. Definition. Let

$$
\begin{gathered}
M_{t}(E, r, x)=\mathrm{M}\left(\Delta\left(S^{n-1}(x, t), \overline{\mathbf{B}}^{n}(x, r) \cap E ; \overline{\mathbb{R}}^{n}\right)\right), \\
M(E, r, x)=M_{2 r}(E, r, x)
\end{gathered}
$$


whenever $E \subset \overline{\mathbb{R}}^{n}, x \in \mathbb{R}^{n}$ and $0<r<t$. We define

$$
\begin{aligned}
& \operatorname{cap} \underline{\operatorname{dens}}(E, x)=\liminf _{r \rightarrow 0} M(E, r, x), \\
& \operatorname{cap} \overline{\operatorname{dens}}(E, x)=\limsup _{r \rightarrow 0} M(E, r, x),
\end{aligned}
$$

where cap $\underline{\operatorname{dens}}(E, x)$ is called the lower and cap $\overline{\operatorname{dens}}(E, x)$ the upper capacity density of $E$ at $x$.

It is usually difficult to calculate or directly estimate the capacity density of a given set $E$. Hence, we introduce another related definition which is useful in obtaining lower bounds for the capacity density.

2.21. Definition. Let $x \in \mathbb{R}^{n}, E \subset \mathbb{R}^{n}$. If the set $A_{x}=\left\{r>0: S^{n-1}(x, r) \cap\right.$ $E \neq \emptyset\}$ is measurable we define the lower and upper radial densities of $E$ at $x$, respectively, by

$$
\begin{aligned}
& \operatorname{rad} \underline{\operatorname{dens}}(E, x)=\liminf _{r \rightarrow 0} \frac{m_{1}\left(A_{x} \cap(0, r)\right)}{r}, \\
& \operatorname{rad} \overline{\operatorname{dens}}(E, x)=\limsup _{r \rightarrow 0} \frac{m_{1}\left(A_{x} \cap(0, r)\right)}{r},
\end{aligned}
$$

where $m_{1}$ is Lebesgue measure on $\mathbb{R}$.

The relationship between capacity and radial densities is given by the following lemma.

2.22. Lemma. [Vuo80, Lemma 2.2.] Let $E$ be an open or compact subset of $\mathbb{R}^{n}$ with $0 \in \bar{E}$ and let $A=\left\{r>0: S^{n-1}(r) \cap E \neq \emptyset\right\}$. Then there exists $c_{n}>0$ depending only on $n$ such that for each $r>0$

$$
M(E, r, 0) \geq c_{n} \log \frac{2 r}{2 r-m_{1}(A \cap(0, r))} .
$$

In particular, if $\operatorname{rad} \underline{\operatorname{dens}}(E, 0)=\delta$, then

$$
\operatorname{cap} \underline{\operatorname{dens}}(E, 0) \geq c_{n} \log \frac{2}{2-\delta} .
$$

2.23. Remark. The constant $c_{n}$ in 2.22 has an expression $c_{n}=2^{n} b_{n}$, where $b_{n}$ is the constant from the spherical cap inequality. The number $b_{n}>0$ has the following expression

$$
\left\{\begin{array}{l}
b_{n}=2^{1-2 n} \omega_{n-2} I_{n}^{1-n}, \quad b_{2}=\frac{1}{2 \pi} \\
I_{n}=\int_{0}^{\pi / 2} \sin ^{\frac{2-n}{n-1}} t d t
\end{array}\right.
$$

See also [Väi71, 10.2 and 10.9.] and [Vuo88, 5.28. and 5.30.]. 
2.24. Quasiregular mappings. A mapping $f: G \rightarrow \mathbb{R}^{n}, n \geq 2$, of a domain $G$ in $\mathbb{R}^{n}$ is called quasiregular if $f$ is in $\mathrm{ACL}^{n}$, and there exists a constant $K$, $1 \leq K<\infty$ such that (see [Ric93], pages 10-11)

$$
\left|f^{\prime}(x)\right|^{n} \leq K J_{f}(x), \quad\left|f^{\prime}(x)\right|=\max _{|h|=1}\left|f^{\prime}(x) h\right|,
$$

a.e. in $G$, where $f^{\prime}(x)$ is the formal derivative. The smallest $K \geq 1$ for which this inequality is true is called the outer dilatation of $f$ and denoted by $K_{O}(f)$. If $f$ is quasiregular, then the smallest $K \geq 1$ for which the inequality

$$
J_{f}(x) \leq K l\left(f^{\prime}(x)\right)^{n}, \quad l\left(f^{\prime}(x)\right)=\min _{|h|=1}\left|f^{\prime}(x) h\right|,
$$

holds a.e. in $G$ is called the inner dilatation of $f$ and denoted by $K_{I}(f)$. The maximal dilatation of $f$ is the number $K(f)=\max \left\{K_{I}(f), K_{O}(f)\right\}$. If $K(f) \leq K, f$ is said to be $K$-quasiregular. A nonconstant quasiregular mapping is discrete and open. A quasiregular homeomorphism $f: G \rightarrow f G$ is called quasiconformal.

The following definitions for $K_{I}(f), K_{O}(f)$ are given in [Väi71, p.41] for a homeomorphism $f: G \rightarrow G^{\prime}$. For a sense-preserving homeomorphism $f$ these definitions coincide with the ones given above:

$$
K_{I}(f)=\sup \frac{\mathrm{M}(f \Gamma)}{\mathrm{M}(\Gamma)}, K_{O}(f)=\sup \frac{\mathrm{M}(\Gamma)}{\mathrm{M}(f \Gamma)},
$$

where the suprema are taken over all path families $\Gamma$ in $G$ such that $\mathrm{M}(\Gamma)$, $\mathrm{M}(f \Gamma)$ are not simultaneously 0 or $\infty$.

The following lemma gives a definition of quasiconformality in terms of the modulus of the path family. This so-called geometric definition is extensively used in the literature. This geometric characterization gives an important method for studying the quasiconformal mappings, and it has many applications.

2.26. Lemma. [Väi71, Theorem 34.3.] A homeomorphism $f: G \rightarrow G^{\prime}$ is quasiconformal if and only if

$$
\mathrm{M}(\Gamma) / K_{O}(f) \leq \mathrm{M}(f(\Gamma)) \leq K_{I}(f) \mathrm{M}(\Gamma)
$$

for every path family $\Gamma$ in $G$.

2.27. Multiplicity and normal domains. Let $f: G \rightarrow \mathbb{R}^{n}$ be a quasiregular mapping. We denote by $i(x, f)$ the infimum of $\sup _{y} \operatorname{card} f^{-1}(y) \cap U$ where $U$ runs through the neighborhoods of $x$. The number $i(x, f)$ is called the local (topological) index of $f$ at $x$. For $r>0$ and $a \in \mathbb{R}^{n}$, the counting function $n(r, a)$ is defined by

$$
n(r, a)=\sum_{x \in f^{-1}(a) \cap \mathbf{B}^{n}(r)} i(x, f) .
$$


Let $f: G \rightarrow \mathbb{R}^{n}$ be a discrete and open mapping. A domain $D \subset \subset G$ is called a normal domain for $f$ if $f \partial D=\partial f D$. A normal neighborhood of $x$ is a normal domain $D$ such that $D \cap f^{-1}(f(x))=\{x\}$.

\section{Consequences of P. JÄrvi's Results}

In this section we study the influence of the local topological index $i(x, f)$ on the behavior of a quasiregular mapping following a recent paper by Järvi [Jär00]. Applying Järvi's results we give a new proof of a result of Rickman and Srebro [RS86] type. Järvi's results presented here will have several applications in the later sections.

Järvi's result was motivated by the following question:

3.1. Question. [Vuo85, 3.8], [Vuo88, p. 193] Let $n \geq 2, K \geq 1$, and $\delta \in(0,1)$. Does there exist a number $d_{0}=d_{0}(n, K, \delta)$ such that if $f: \mathbf{B}^{n} \rightarrow \mathbf{B}^{n}$ is a nonconstant $K$-quasiregular mapping with $|f(0)| \geq \delta$, then $\operatorname{card} Z \leq d_{0}$, where $Z=\left\{x \in \mathbf{B}^{n}:|x| \leq 1 / 2, f(x)=0\right\} ?$

In order to answer the question Järvi proved the following theorem, which yields the solution to 3.1 with

$$
d_{0}(n, K, \delta)=d_{1}\left[(\log 2)^{-3} \log (1 / \delta)\right]^{n-1} \exp \left(d_{2}(\log 2)^{(2-2 n) / n}\right),
$$

where constants $d_{1}>0$ and $d_{2}>0$ depend only on $n, K$. (See Corollary 3.7 and Theorem 3.12 for details.)

3.2. Theorem. [Jär00, Theorem 3.8] Let $f: \mathbf{B}^{n} \rightarrow \mathbf{B}^{n}$ be a K-quasiregular mapping. Then there is a constant $d_{3} \in(0,1)$ depending only on $n$ and $K$ such that

$$
|f(x)| \leq \exp \left(-d_{3} \int_{\frac{2}{3}|x|}^{\frac{1}{2}} \frac{n(t, 0)^{1 /(n-1)}}{t} d t\right) \quad \text { for all } x \in \mathbf{B}^{n} .
$$

The constant $d_{3}$ has the following expression

$$
d_{3}=\frac{\left(K_{I}^{2} K_{O}\right)^{1 /(1-n)}}{d_{4}},
$$

where $d_{4}>1$ is the constant in the Harnack inequality (setting $r=3 / 2$ and $R=2$ in [Ric93, Theorem VI.7.4]) depending only on $n$ and $K$.

3.3. Schwarz lemma. The Schwarz lemma states that if $f: \mathbf{B}^{2} \rightarrow \mathbf{B}^{2}$ is analytic, and $f(0)=0$, then $|f(z)| \leq|z|$ for $z \in \mathbf{B}^{2}$. The counterpart of the Schwarz lemma for quasiregular maps $f: \mathbf{B}^{n} \rightarrow \mathbf{B}^{n}$ with $f(0)=0$ is given in [Ric93, III.1.10.], [Vuo88, 11.2.].

The next result can be regarded as a refined version of this result involving the local topological index $i(0, f)$. The idea here is that the estimate becomes better as $i(0, f)$ grows. In fact, it is possible to show [Car50, 141.] that for an analytic function $f: \mathbf{B}^{2} \rightarrow \mathbf{B}^{2}$ with $f(0)=0$ and $i(0, f)=p>1$ we have 
$|f(z)| \leq|z|^{p}$ for all $z \in \mathbf{B}^{2}$. Theorem 3.4 is a counterpart of this result for quasiregular mappings in $\mathbb{R}^{n}$.

3.4. Theorem. [Jär00, 3.9.] Let $f: \mathbf{B}^{n} \rightarrow \mathbf{B}^{n}$ be a $K$-quasiregular mapping with $f(0)=0$. Then

$$
|f(x)| \leq d_{5}|x|^{\mu} \exp \left(-d_{3} \int_{\frac{2}{3}|x|}^{\frac{1}{2}} \frac{n(t, 0)^{1 /(n-1)}-i(0, f)^{1 /(n-1)}}{t} d t\right)
$$

for all $x \in \mathbf{B}^{n}$, where $d_{3} \in(0,1)$, is a constant depending only on $n, K$ as in Theorem 3.2, $d_{5}=(4 / 3)^{\mu}$ and $\mu=d_{3} i(0, f)^{1 /(n-1)}$. In particular, $|f(x)| \leq$ $(2 / 3)^{\mu}$ for all $|x| \leq 1 / 2$.

Previously, a result similar to the second part of Theorem 3.4 was known to hold only locally, in a neighborhood of 0 , but nothing could be said about the size of this neighborhood. See e.g. [Ric93, Theorem III.4.7] and the references given in [Ric93, III.4.9].

Theorem 3.2 gives a new result of the same type as the main result of S. Rickman and U. Srebro in [RS86].

3.5. Theorem. Let $n \geq 2$ be an integer and $K \geq 1$. Let $d_{3} \in(0,1)$ be as in Theorem 3.2, and let $f: G \rightarrow \mathbf{B}^{n}$ be a nonconstant $K$-quasiregular mapping. Fix $x_{0} \in G$ with $\mu \equiv d_{3} i\left(x_{0}, f\right)^{1 /(n-1)}>1$ and choose $t_{0} \in\left(0, d\left(x_{0}, \partial G\right) / 2\right)$ such that $\overline{\mathbf{B}}^{n}\left(x_{0}, t_{0}\right)$ is a subset of a normal neighborhood of $x_{0}$.

Then there exist integers $m>2$ and $j \in\{1, \ldots, m\}$, such that if $x_{1}, \ldots, x_{m} \in \overline{\mathbf{B}}^{n}\left(x_{0}, t_{0}\right)$ with $\left|x_{0}-x_{m}\right| \geq t_{0}>0$ and $\left|x_{k-1}-x_{k}\right|<10 t_{0} / m$ for all $k=1, \ldots, m$, then $i\left(x_{j}, f\right)<i\left(x_{0}, f\right)$.

Proof. Suppose that this is not the case. Let $x_{1}, \ldots, x_{m}$ be as the points in the theorem with $i\left(x_{j}, f\right) \geq i\left(x_{0}, f\right)>1$ for all $j=1, \ldots, m$. For each $x_{j}$, we define a quasiregular mapping $g_{j}$ by $g_{j}(z)=\frac{1}{2}\left(f\left(t_{0} z+x_{j}\right)-f\left(x_{j}\right)\right)$. We note that $g_{j}: \mathbf{B}^{n} \rightarrow \mathbf{B}^{n}$ with $g_{j}(0)=0$ and $x_{j}+t_{0} z \in G$ for $z \in \mathbf{B}^{n}$. By applying the estimate of Theorem 3.4 to each $g_{j}$, we may conclude that

$$
\left|f\left(x_{j-1}\right)-f\left(x_{j}\right)\right| \leq 2 d_{5}\left|\frac{x_{j-1}-x_{j}}{t_{0}}\right|^{\mu}
$$


where $d_{5}$ is as in Theorem 3.4. Then by the choice of $t_{0}$

$$
\begin{aligned}
0 & <d\left(f\left(x_{0}\right), f S^{n-1}\left(x_{0}, t_{0}\right)\right) \\
& \leq\left|f\left(x_{0}\right)-f\left(x_{m}\right)\right| \\
& \leq \sum_{j=1}^{m}\left|f\left(x_{j}\right)-f\left(x_{j-1}\right)\right| \\
& \leq \sum_{j=1}^{m} 2 d_{5} \frac{\left|x_{j}-x_{j-1}\right|^{\mu}}{t_{0}^{\mu}} \\
& \leq 2 d_{5} m \frac{\left|10 t_{0} / m\right|^{\mu}}{t_{0}^{\mu}} \\
& \leq 2 \cdot(40 / 3)^{\mu} d_{5} m^{1-\mu}
\end{aligned}
$$

This is a contradiction, if $m$ is large enough, as $\mu>1$ and $d\left(f\left(x_{0}\right), f S^{n-1}\left(x_{0}, t_{0}\right)\right)>0$ does not depend on $m$.

Let $R>0$ and let $f: \mathbf{B}^{n}(R) \rightarrow \mathbb{R}^{n}$ be quasiregular. For $r \in(0, R)$, we set

$$
M(r, f)=M(r)=\max \left\{|f(x)|: x \in S^{n-1}(r)\right\} .
$$

Furthermore, if $f$ is bounded, we define

$$
M(R)=\sup \left\{|f(x)|: x \in \mathbf{B}^{n}(R)\right\} .
$$

3.6. Lemma. [Jär00, Lemma 3.1.] Let $r>0$ and let $f: \mathbf{B}^{n}(2 r) \rightarrow \mathbb{R}^{n}$ be a bounded $K$-quasiregular mapping. Then there is a constant

$d_{7}=d_{8}\left(\omega_{n-1} / K_{I}\right)^{1 /(n-1)} \in(0,1)$ depending only on $n$ and $K$ such that

$$
\frac{M\left(\frac{3}{2} r\right)}{M(2 r)} \leq \exp \left(-d_{7} n(r, 0)^{1 /(n-1)}\right),
$$

where $d_{8} \in(0,1)$ is the constant in [Ric93], Lemma VII.4.7 depending only on $n$ and $K$.

3.7. Corollary. [Jär00, Corollary 3.6.] Let $f: \mathbf{B}^{n} \rightarrow \mathbf{B}^{n}$ be a $K$-quasiregular mapping with $|f(0)| \geq \delta>0$. Then $n\left(\frac{1}{2}, 0\right) \leq\left(\frac{1}{d_{7}} \log \frac{1}{\delta}\right)^{n-1}$, where $d_{7}>0$ is the constant in Lemma 3.6.

3.8. Lemma. [Väi71, Example 16.2.] Let $s \in(0,1)$ and let $h: \overline{\mathbf{B}}^{n} \rightarrow \overline{\mathbf{B}}^{n}$ be the mapping

$$
h(x)= \begin{cases}|x|^{\psi-1} x & \text { if } x \in \overline{\mathbf{B}}^{n} \backslash\{0\} \\ 0 & \text { if } x=0\end{cases}
$$

where $\psi=\frac{\log 2}{\log \frac{1}{s}}$. Then $h$ is quasiconformal and $K(h) \leq \max \left\{\psi^{n-1}, \psi^{1-n}\right\}$.

3.9. Variational kernels. Let $W^{1, n}(U)$ denote the Sobolev space consisting of all real valued functions $u$ in $L^{n}(U)$ with weak first order partial derivatives 
also in $L^{n}(U)$. Here $U$ is an open set in $\mathbb{R}^{n}$. The closure of $\mathcal{C}_{0}^{\infty}(U)$ in $W^{1, n}(U)$ is denoted by $W_{0}^{1, n}(U)$ (see e.g. [Ric93, pp. 5-7]).

Let $F: U \times \mathbb{R}^{n} \rightarrow \mathbb{R}$ be a variational kernel (see [Ric93, VI.1.3]). Let $v \in$ $W^{1, n}(U)$. We say that $u \in W^{1, n}(U)$ is $F$-extremal for boundary values $v$ if

(1) $u-v \in W_{0}^{1, n}(U)$ and

$$
\int_{U} F(x, \nabla u(x)) d x \leq \int_{U} F(x, \nabla w(x)) d x
$$

for all $w \in W^{1, n}(U)$ with $w-v \in W_{0}^{1, n}(U)$.

A function $u \in W_{l o c}^{1, n}(U)$ is a (free) F-extremal if $u \mid V$ is $F$-extremal for the boundary values $u \mid \partial V$ whenever $V \subset \subset U$.

3.10. Lemma. Let $G \subset \mathbb{R}^{n}$ be a domain, and let $f: G \rightarrow \mathbb{R}^{n}$ be a nonconstant quasiregular mapping. Set $F_{I}(x, h)=|h|^{n}$ and let $f^{\#} F_{I}: G \times \mathbb{R}^{n} \rightarrow \mathbb{R}$ denote the pullback kernel of $F_{I}$ with respect of $f$. Then $f^{\#} F_{I}$ satisfies the inequality

$$
\alpha|h|^{n} \leq f^{\#} F_{I}(x, h) \leq \beta|h|^{n}
$$

for all $h \in \mathbb{R}^{n}$ a.e. $x \in G$ with $\alpha=K_{O}(f)^{-1}$ and $\beta=K_{I}(f)$.

For the proof and discussion, see [Ric93], pages 130 and 135-136.

Let $C \subset U$ be compact. If $\varphi$ is a function in $\mathcal{C}_{0}^{\infty}(U)$ such that $0 \leq \varphi \leq 1$ and $\varphi \mid V=1$ for some neighborhood $V$ of $C$, we let $u \in W^{1, n}(U \backslash C) \cap \mathcal{C}(U \backslash C)$ be the $F$-extremal with boundary values $\varphi$. Then the function $u$ is independent of the choice of $\varphi$ and $0 \leq u \leq 1$. We call $u$ the $F$-potential of the condenser $(U, C)$. The integral

$$
\int_{U \backslash C} F(\cdot, \nabla u) d m
$$

is called the $F$-capacity of $(U, C)$ and denoted by $\operatorname{cap}_{F}(U, C)$.

3.11. An upper bound for multiplicity. In the statement of Problem 3.1 the role of the constant $1 / 2$ is not very important. Next we show how Järvi's solution to Problem 3.1 can be modified in order to establish a result where $1 / 2$ is replaced by an arbitrary $s \in(1 / 2,1)$.

3.12. Theorem. Let $f: \mathbf{B}^{n} \rightarrow \mathbf{B}^{n}$ be a K-quasiregular mapping with $|f(0)| \geq$ $\delta>0$ and $1 / 2 \leq s<1$. Then

$$
n(s, 0) \leq d_{1}\left[\left(\log \frac{1}{s}\right)^{-3} \log \frac{1}{\delta}\right]^{n-1} \exp \left(d_{2}\left(\log \frac{1}{s}\right)^{(2-2 n) / n}\right),
$$

where the constants $d_{1}>0$ and $d_{2}>0$ depend only on $K$ and $n$.

Proof. Let $g=f \circ h^{-1}$, where $h$ is as in Lemma 3.8. Then $g$ is quasiregular and $K(g) \leq K(f) K\left(h^{-1}\right)$. Clearly $g$ satisfies the conditions of Corollary 3.7. 
Let $r>0, C \subset \mathbf{B}^{n}(r), V=\mathbf{B}^{n}(2 r)$ and let $u$ be the $g^{\#} F_{I}$-potential of the condenser $(V, C)$, as in [Ric93, VII.4.7]. By Lemma $3.10 g^{\#} F_{I}$ satisfies the conditions of [Ric93, VII.4.7], yielding the estimate

$$
u(x) \geq d_{8} \operatorname{cap}(V, C)^{1 /(n-1)}
$$

for $x \in \overline{\mathbf{B}}^{n}(3 r / 2) \backslash C$. We study the constant $d_{8} \in(0,1)$ in (3.13) which is the same as the constant $d_{8}$ in Lemma 3.6. The proof of this lower bound gives for each $\varepsilon>0$ also the inequality

$$
\alpha \operatorname{cap}(V, C) \leq \beta d_{4}^{n-1}(m+\varepsilon)^{n-1} \operatorname{cap}\left(V, \overline{\mathbf{B}}^{n}\left(\frac{3 r}{2}\right)\right)
$$

where $\alpha=K_{O}(g)^{-1}, \beta=K_{I}(g), m=\min _{S^{n-1}(3 r / 2)} u>0$, and $d_{4}>1$ is the constant in Harnack inequality for nonnegative continuous $F$-extremals, [Ric93, Theorem VI.7.4]. The constant $d_{4}$ has the representation

$$
d_{4}=\exp \left[2\left(b_{n}^{-1}\left(\frac{n}{n-1}\right)^{n} \beta \alpha^{-1} \omega_{n-1}\right)^{1 / n}\left(\log \frac{4}{3}\right)^{-1}\right],
$$

where $b_{n}>0$ is as in Remark 2.23. It follows that

$$
\left(\frac{\alpha}{\beta}\right)^{1 /(n-1)} \operatorname{cap}(V, C)^{1 /(n-1)} \leq d_{4}(m+\varepsilon) \operatorname{cap}\left(V, \overline{\mathbf{B}}^{n}\left(\frac{3 r}{2}\right)\right)^{1 /(n-1)},
$$

and hence

$$
\left(\frac{\alpha}{\beta}\right)^{1 /(n-1)}\left[\operatorname{cap}\left(V, \overline{\mathbf{B}}^{n}\left(\frac{3 r}{2}\right)\right)^{1 /(n-1)} d_{4}\right]^{-1} \operatorname{cap}(V, C)^{1 /(n-1)} \leq m+\varepsilon .
$$

By Lemma 2.10

$$
\operatorname{cap}\left(V, \overline{\mathbf{B}}^{n}\left(\frac{3 r}{2}\right)\right)=\operatorname{cap}\left(\mathbf{B}^{n}(2 r), \overline{\mathbf{B}}^{n}\left(\frac{3 r}{2}\right)\right)=\omega_{n-1}\left(\log \frac{4}{3}\right)^{1-n} .
$$

Since by [Ric93, VI.7.16] $u(x) \geq m$ for $x \in \mathbf{B}^{n}(3 r / 2) \backslash C$,

$$
d_{8}=\left(\frac{\alpha}{\beta}\right)^{1 /(n-1)}\left(\log \frac{4}{3}\right) d_{4}^{-1} \omega_{n-1}^{1 /(1-n)} .
$$

Now we get the estimate for the constant $d_{7}$ in Lemma 3.6,

$$
d_{7}^{1-n} \leq\left(\log \frac{4}{3}\right)^{1-n} K_{I}(g)^{2} K_{O}(g) \exp \left(E_{n}\left(K_{I}(g) K_{O}(g)\right)^{1 / n}\right)
$$

where

$$
E_{n}=2\left(b_{n}^{-1} \omega_{n-1}\right)^{1 / n}\left(\frac{n}{n-1}\right)\left(\log \frac{4}{3}\right)^{-1} .
$$

By the fact that

$$
\max \left\{K_{I}(g), K_{O}(g)\right\}=K(g)=K\left(f \circ h^{-1}\right) \leq\left(\frac{\log \frac{1}{s}}{\log 2}\right)^{1-n} K(f),
$$


we may conclude

$$
d_{7}^{1-n} \leq\left(\log \frac{4}{3}\right)^{1-n} K(f)^{3}\left(\frac{\log \frac{1}{s}}{\log 2}\right)^{3(1-n)} \exp \left(E_{n} K(f)^{2 / n}\left(\frac{\log \frac{1}{s}}{\log 2}\right)^{(2-2 n) / n}\right) .
$$

By defining

$$
d_{1}=K(f)^{3}\left(\log \frac{4}{3}\right)^{1-n}(\log 2)^{3(n-1)}, d_{2}=K(f)^{2 / n} E_{n}(\log 2)^{(2 n-2) / n},
$$

and by applying Corollary 3.7 we obtain

$$
n(s, 0) \leq d_{1}\left[\left(\log \frac{1}{s}\right)^{-3} \log \frac{1}{\delta}\right]^{n-1} \exp \left(d_{2}\left(\log \frac{1}{s}\right)^{(2-2 n) / n}\right) .
$$

\section{Applications of tWo-COnstants theorem}

In this section, the main idea is to combine Rickman's two-constants theorem with the results of Järvi from Section 3. This allows us to extend the estimates given by Järvi's results to hold in the whole domain of definition. The results proven here will be applied later in Section 5.

In Rickman's two-constants theorem we consider a quasiregular mapping, $f: G \rightarrow \mathbb{R}^{n}$ in two concentric balls, not necessarily contained in $G$, and obtain a growth estimate for the mapping $f$. This result plays a central role in Rickman's proof of his version of Picard's theorem for quasiregular mappings in $\mathbb{R}^{n}$.

4.1. Theorem. [Ric80a, Theorem 4.22.] Let $f: G \rightarrow \mathbb{R}^{n}$ be a nonconstant $K$-quasiregular mapping, let $0<m<M$, and suppose

(1) $|f(x)| \leq M$ if $x \in G \cap \mathbf{B}^{n}(z, s)$,

(2) $\lim \sup _{x \rightarrow y}|f(x)| \leq m$ if $y \in \partial G \cap \overline{\mathbf{B}}^{n}(z, s)$.

Then there exist $r_{0} \in(0,1 / 2), C \in\left(0, \omega_{n-1}^{1 /(1-n)}\right)$ depending only on $n$ and $K$ such that for all $r \in\left(0, r_{0}\right)$ and all $x \in \mathbf{B}^{n}(z, r s) \cap G$

$$
|f(x)| \leq \exp (\beta \log m+(1-\beta) \log M),
$$

where

$$
\beta=C \operatorname{cap}\left(\mathbf{B}^{n}(z, s), \overline{\mathbf{B}}^{n}(z, r s) \backslash G\right)^{1 /(n-1)} \log (1 / r) \in[0,1) .
$$

From the two-constants theorem one may derive a version in which the function is bounded with a constant $\varepsilon$ on a compact subset $F$ of $G$, and a bound is obtained in the whole domain $G$. This version is due to M. Vuorinen.

The bound involves the quasihyperbolic distance $k_{G}(x, y)$ between two points $x, y \in G$, see e.g. [Vuo88, p.33]. It is well-known that $k_{\mathbf{H}^{n}}=\rho_{\mathbf{H}^{n}}$ and $\rho_{\mathbf{B}^{n}} \leq$ $2 k_{\mathbf{B}^{n}} \leq 2 \rho_{\mathbf{B}^{n}}$. 
4.2. Lemma. [Vuo81, Lemma 2.22.] Let $f: G \rightarrow \mathbf{B}^{n}$ be $K$-quasiregular, $F \subset G$ compact, $k_{G}(F) \leq 2, \operatorname{cap}(G, F) \geq \delta>0$, and $|f(x)| \leq \varepsilon$ for $x \in F$. Then there is a number $\beta \in(0,1)$ depending only on $n, K, \delta$ and $d(F) / d(F, \partial G)$ such that

$$
|f(y)| \leq \varepsilon^{A} ; A=\beta^{\left(1+d_{6} k_{G}(y, F)\right)},
$$

for $y \in G$ and $d_{6}=1 / \log \left(1+\lambda_{K}\right)>1$, where $\lambda_{K} \in(0,1 / 2)$ is a constant depending only on $n$ and $K$.

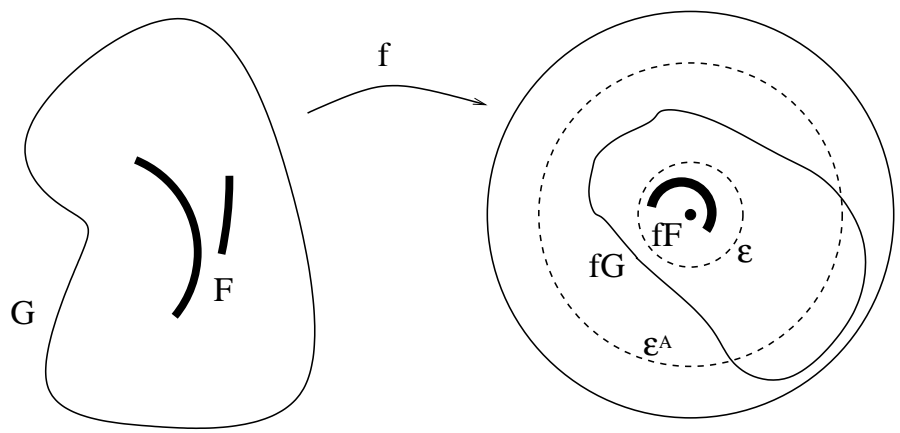

Figure 1. The mapping $f$ of Lemma 4.2.

4.3. Lemma. Let $r \in(0,1 / 2]$, and let $f: \mathbf{B}^{n} \rightarrow \mathbf{B}^{n}$ be a $K$-quasiregular mapping with $f(0)=0$. Then

$$
|f(y)| \leq\left(\frac{4}{3} r\right)^{\mu A} ; A=\beta^{1+d_{6} \rho(y, 0)} \in(0,1)
$$

for $y \in \mathbf{B}^{n}$, where $\mu=d_{3} i(0, f)^{1 /(n-1)}, d_{3} \in(0,1), d_{6}>1$ are constants depending only on $n, K$ as in Lemma 4.2 and $\beta=\beta(r, n, K) \in(0,1)$.

Proof. By Theorem 3.4,

$$
|f(x)| \leq\left(\frac{4|x|}{3}\right)^{\mu}
$$

for $x \in \mathbf{B}^{n}(r)$, and by applying Lemma 4.2 with $G=\mathbf{B}^{n}$ and $F=\overline{\mathbf{B}}^{n}(r)$, we have

for $y \in \mathbf{B}^{n}$.

$$
|f(y)| \leq\left(\frac{4 r}{3}\right)^{\mu A} ; A=\beta^{1+d_{6} \rho(0, y)}
$$

4.4. Sequential limits. Next assume that a mapping $f$ has a limit along a sequence of points tending to a point in the boundary. We try to find conditions under which the mapping has a limit in a larger set containing the sequence.

We observe that for a continuous $f: \mathbf{B}^{n} \rightarrow \mathbb{R}^{n}, b_{k} \in \mathbf{B}^{n}, b_{k} \rightarrow b \in \partial \mathbf{B}^{n}$, $f\left(b_{k}\right) \rightarrow \beta$ and $\beta \in \overline{\mathbb{R}}^{n}$, it is easy to see that there is an open set $E$, with $b_{k} \in E, b \in \bar{E}$ such that $f(x) \rightarrow \beta$ when $x \in E, x \rightarrow b$. 
It should be noted that if $b_{k} \rightarrow b \in \partial \mathbf{B}^{n}$ and $\lim f\left(b_{k}\right)=\beta$, it does not necessarily follow that $f$ has a limit along the set $E=\bigcup_{k=1}^{\infty} D\left(b_{k}, m\right)$ for $m>0$. To see this consider the map $f: \mathbf{B}^{2} \rightarrow \mathbf{B}^{2} \backslash\{0\}$ defined by formula $f(z)=$ $\exp (g(z))$, where $g(z)=-(1+z) /(1-z)$ for $z \in \mathbf{B}^{2}$. Fix $\beta \in \mathbf{B}^{2} \backslash\{0\}$ and let $b_{k} \in \mathbf{B}^{2}, b_{k} \rightarrow 1$ with $\lim f\left(b_{k}\right)=\beta$. Then there is $m>0$ such that $f D\left(b_{k}, m\right)$ is a set independent of $k$. This example shows that in the next result it is essential that $\mu_{k}=d_{3} i\left(z_{k}, f\right)^{1 /(n-1)} \rightarrow \infty$ as $k \rightarrow \infty$.

4.5. Lemma. Let $\left(z_{k}\right)$ be a sequence of points in $\mathbf{H}^{n}$ with $z_{k}=\left|z_{k}\right| e_{n}$ and $z_{k} \rightarrow$ 0 . Let $f: \mathbf{H}^{n} \rightarrow \mathbf{B}^{n}$ be $K$-quasiregular with $f\left(z_{k}\right)=0$, let $d_{3}, \beta=\beta(1 / 2, n, K)$, $d_{6}$ be as in Lemma 4.3 and let $\mu_{k} \equiv d_{3} i\left(z_{k}, f\right)^{1 /(n-1)} \rightarrow \infty$. If

$$
M_{k}=\frac{\log \mu_{k}}{2 d_{6} \log \frac{1}{\beta}},
$$

and $E=\bigcup_{k=1}^{\infty} D\left(z_{k}, M_{k}\right)$, then $f(x) \rightarrow 0$ as $x \rightarrow 0, x \in E$.

Proof. Let $r=1 / 2$. Let $g_{k}$ be a Möbius transformation with $g_{k}\left(\mathbf{B}^{n}\right)=\mathbf{H}^{n}$ and $g_{k}(0)=z_{k}$. Then by Lemma 4.3

$$
\left|f \circ g_{k}(y)\right| \leq(2 / 3)^{\mu_{k} A}
$$

for $y \in \mathbf{B}^{n}$, where $A$ is as in Lemma 4.3 .

We need to find $M_{k}$ such that for $\rho\left(y, z_{k}\right) \leq M_{k}$,

$$
\mu_{k} \beta^{1+d_{6} M_{k}} \rightarrow \infty
$$

This holds for

$$
\beta^{d_{6} M_{k}}=\frac{1}{\sqrt{\mu_{k}}}
$$

which is equivalent to

$$
M_{k}=\frac{\log \mu_{k}}{2 d_{6} \log \frac{1}{\beta}}
$$

4.6. Lemma. Let $D_{M}=D\left(e_{n}, M\right)$ and $V_{M}=\mathbf{B}_{+}^{n} \backslash D_{M}$. Then

$$
\frac{m\left(V_{M}\right)}{m\left(\mathbf{B}_{+}^{n}\right)} \leq \frac{2 \Omega_{n-1}}{\Omega_{n}} \frac{1}{\cosh M}
$$

In particular,

$$
\frac{m\left(V_{M}\right)}{m\left(\mathbf{B}_{+}^{n}\right)} \rightarrow 0 \text { as } M \rightarrow \infty
$$

Proof. Let $s_{M}=d\left(\partial D_{M} \cap S^{n-1}, \partial \mathbf{H}^{n}\right)$, as in Figure 2 . 


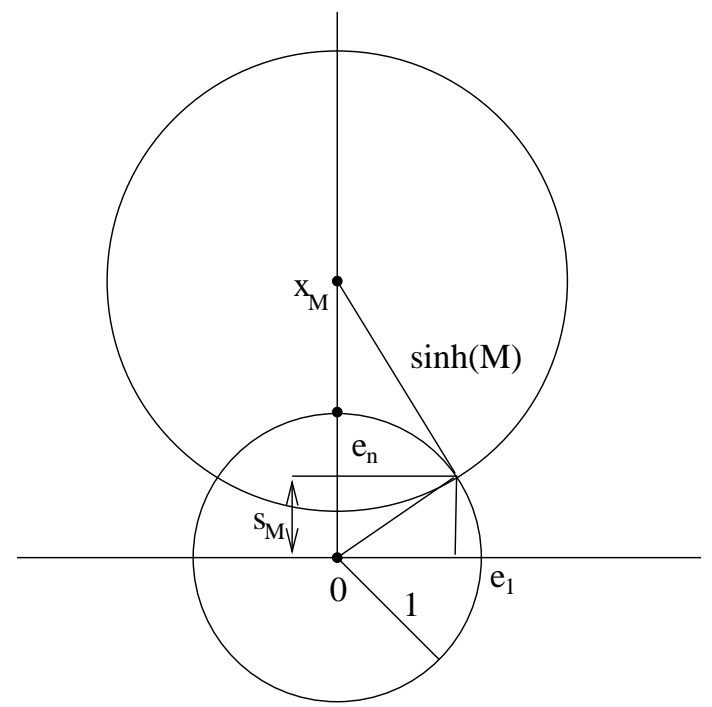

Figure 2. Ball $D_{M}$ of Lemma 4.6.

By (2.4) we have

$$
D\left(e_{n}, M\right)=\mathbf{B}^{n}\left((\cosh M) e_{n}, \sinh M\right) .
$$

By similar triangles we obtain the equality

$$
s_{M}=\frac{1}{\cosh M} .
$$

Because $m\left(V_{M}\right) \leq s_{M} \Omega_{n-1}$ and $m\left(\mathbf{B}_{+}^{n}\right)=\Omega_{n} / 2$, we have

$$
0 \leq \frac{m\left(V_{M}\right)}{m\left(\mathbf{B}_{+}^{n}\right)} \leq s_{M} \frac{2 \Omega_{n-1}}{\Omega_{n}} .
$$

We conclude that

$$
\frac{m\left(V_{M}\right)}{m\left(\mathbf{B}_{+}^{n}\right)} \leq \frac{2 \Omega_{n-1}}{\Omega_{n}} \frac{1}{\cosh M}
$$

and thus $s_{M} \rightarrow 0$ as $M \rightarrow \infty$.

4.7. Measure densities. Let $E \subset \mathbb{R}^{n}$ be a measurable set and $x \in \mathbb{R}^{n}$. The upper measure density of $E$ at $x$ is defined to be

$$
\theta^{n *}(E, x)=\limsup _{r \rightarrow 0} \frac{m\left(E \cap \overline{\mathbf{B}}^{n}(x, r)\right)}{\Omega_{n} r^{n}}
$$

where $\Omega_{n}=m\left(\mathbf{B}^{n}\right)$ and the lower measure density $\theta_{*}^{n}(E, x)$ is the corresponding liminf. If $\theta^{n *}(E, x)=\theta_{*}^{n}(E, x)$, this common value is the measure density $\theta^{n}(E, x)$ of $E$ at $x$. 
4.8. Lemma. Let $z_{k} \in\left(0, e_{n}\right),\left|z_{k}\right|>\left|z_{k+1}\right|$ and $\left|z_{k}\right| \rightarrow 0$. Let $f: \mathbf{H}^{n} \rightarrow \mathbf{B}^{n}$ be a $K$-quasiregular mapping with $f\left(z_{k}\right)=0$, and $\mu_{k}=c_{2} i\left(z_{k}, f\right)^{1 /(n-1)} \rightarrow \infty$. Then there is a set $E$ such that $\left\{z_{k}\right\} \subset E, \lim _{x \rightarrow 0, x \in E} f(x)=0$ and $\theta^{n *}(E, 0)=$ $\theta^{n}\left(\mathbf{H}^{n}, 0\right)$. Furthermore, rad $\overline{\operatorname{dens}}(E, 0)=1$.

Proof. By Lemma 4.5 we find $M_{k}$ such that $\lim _{x \rightarrow 0, x \in E} f(x)=0$ if $E=$ $\bigcup_{k=1}^{\infty} D\left(z_{k}, M_{k}\right)$, and by Lemma 4.6

$$
\limsup _{k \rightarrow \infty} \frac{m\left(E \cap \mathbf{B}_{+}^{n}\left(\left|z_{k}\right|\right)\right)}{m\left(\mathbf{B}_{+}^{n}\left(\left|z_{k}\right|\right)\right)}=1 .
$$

It follows from $(2.2)$ that $\operatorname{rad} \overline{\operatorname{dens}}(E, 0)=1$.

4.9. Remark. An explicit construction for a function satisfying the conditions of Lemma 4.8 is given in Example 5.21.

\section{Multiplicity AND CONVERGENCE}

In this section we use Järvi's method to refine some results of Vuorinen [Vuo85] concerning the speed of convergence of quasiregular mappings to a constant along a sequence of points or continua. In the complex plane similar questions for analytic functions have been studied by D. C. Rung [Run68] and recently by V. Eiderman and M. Essén in [EE02].

In the main results of this section we give criteria for a quasiregular mapping to have an angular limit and a limit along a tangential set. The criteria studied here involve the local topological index of the mapping on a sequence of points approaching the boundary. An example by P. Lappan gives an explicit construction of a mapping in the complex plane with no angular limit and local topological index approaching infinity on a sequence of points.

5.1. Angular limits. If $\varphi \in(0, \pi / 2)$, then we write

$$
C(\varphi)=\left\{z=\left(z_{1}, \ldots, z_{n}\right) \in \mathbf{H}^{n}: z_{n}>|z| \cos \varphi\right\}
$$

Similarly we define the cone at the point $b, C(b, \varphi)$ for $b \in \partial \mathbf{H}^{n}$. For $b \in \partial \mathbf{B}^{n}$, we denote by $C(b, \varphi)$ the cone $\left\{z \in \mathbb{R}^{n}:(b \mid b-z)>|b-z| \cos \varphi\right\}$. Here $(x \mid y)$ is the inner product $\sum_{i=1}^{n} x_{i} y_{i}$.

Let $f: \mathbf{H}^{n} \rightarrow Y$ be a locally integrable function, where $Y=\mathbb{R}^{n}$ or $Y=\mathbb{R}$, and let $\alpha \in Y$. Then the function $f$ is said to have an angular limit $\alpha$ at 0 if for each $\varphi \in(0, \pi / 2), f(x) \rightarrow \alpha$ when $x \rightarrow 0$ and $x \in C(\varphi)$.

If $E$ is a set in $\mathbf{H}^{n}$ with $0 \in \bar{E}$ and $E \subset C(\varphi)$ for some $\varphi \in(0, \pi / 2)$, then we say that $E$ is non-tangential at 0 . Otherwise we say that $E$ is tangential. 


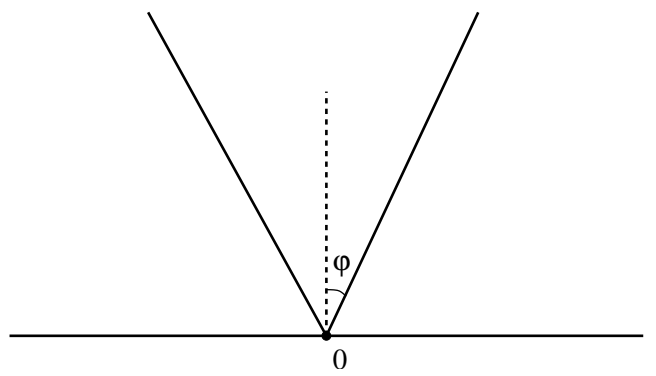

Figure 3. The cone $C(\varphi)$

We need the following two results by Vuorinen to prove the main results.

5.2. Theorem. [Vuo81, Theorem 3.1.] Let $f: \mathbf{B}^{n} \rightarrow \mathbf{B}^{n}$ be a quasiregular mapping, $b \in S^{n-1}, \varphi_{0} \in(0, \pi / 2)$ and let $E \subset C\left(b, \varphi_{0}\right)$. If $f(x)$ tends to 0 when $x$ approaches $b$ through $E$ and if cap dens $(E, b)=\delta>0$ then $f$ has an angular limit 0 at $b$, i.e. $\lim _{x \rightarrow b, x \in C(b, \varphi)} f(x)=0$ for all $\varphi \in(0, \pi / 2)$.

5.3. Lemma. [Vuo85, Lemma 3.2.] For $n \geq 2$ and $K \geq 1$ there exist positive numbers $C$ and $\lambda_{K} \in(0,1 / 2)$ with the following properties. If $f: \mathbf{H}^{n} \rightarrow \mathbf{B}^{n}$ is a bounded $K$-quasiregular mapping, $F$ is a continuum in $\mathbf{H}^{n}$ with $\rho(F) \geq w>0$ and if $|f(y)-\alpha| \leq \varepsilon$ for $y \in F$, then

$$
|f(x)-\alpha| \leq \varepsilon^{A}(1+|\alpha|)^{1-A} ; \quad A=\beta^{1+d_{6} \rho(x, F)}
$$

for all $x \in \mathbf{H}^{n}$, where $\rho$ stands for the hyperbolic metric,

$$
\begin{gathered}
\beta=a_{1}\left(\min \left\{w, \log \left(1+\lambda_{K}\right)\right\}\right)^{1 /(n-1)} \in(0,1) \\
d_{6}=1 / \log \left(1+\lambda_{K}\right)>1 ; \quad a_{1}=C a_{2}
\end{gathered}
$$

and $a_{2}>0$ depends only on $n$.

The next result is closely related to [Vuo85, Theorem 3.6.]. The difference is that the estimates here involve the local topological index $i(x, f)$. This result gives a criterion for a quasiregular mapping to have an angular limit.

5.4. Theorem. Let $f: \mathbf{H}^{n} \rightarrow \mathbf{B}^{n}$ be a K-quasiregular mapping, let $d_{6}, \beta$ be as above, $d_{3}$ as in Theorem 3.2 and let $t_{k}=2^{-k}$ and $f\left(t_{k} e_{n}\right)=0$ for all $k=0,1, \ldots$

(1) If $\lim \sup _{k \rightarrow \infty} t_{k}^{\gamma} \mu\left(t_{k} e_{n}, f\right)=\infty$, where $\mu\left(t_{k} e_{n}, f\right)=d_{3} i\left(t_{k} e_{n}, f\right)^{1 /(n-1)}$ and $\gamma=d_{6} \log (1 / \beta)$, then $f \equiv 0$.

(2) If $\mu\left(t_{k} e_{n}, f\right) \rightarrow \infty$ as $k \rightarrow \infty$, then $f$ has an angular limit 0 at the origin.

Proof. (1) Let $x \in \mathbf{H}^{n}$ with $f(x)=0$ and $r \in\left(0, d\left(x, \partial \mathbf{H}^{n}\right)\right)$. We denote by $f_{x, r}$ the restriction of $f$ to the ball $\mathbf{B}^{n}(x, r)$. We define

$$
g_{x, r}(z)=\frac{1}{2} f_{x, r}(x+r z), \quad z \in \mathbf{B}^{n} .
$$


Now $g_{x, r}: \mathbf{B}^{n} \rightarrow \mathbf{B}^{n}$ is $K$-quasiregular and $g_{x, r}(0)=0$. It follows from Theorem 3.4 , that

$$
\left|g_{x, r}(z)\right| \leq d_{5}|z|^{\mu\left(0, g_{x, r}\right)}=\left(\frac{4}{3}|z|\right)^{\mu\left(0, g_{x, r}\right)} \leq\left(\frac{2}{3}\right)^{\mu\left(0, g_{x, r}\right)}
$$

for $z \in \mathbf{B}^{n}(0,1 / 2)$.

We choose $x=t_{k} e_{n}$ and $r=t_{k}$. By the definition of $g_{x, r}$

$$
\frac{\left|f(w)-f\left(t_{k} e_{n}\right)\right|}{2} \leq\left(\frac{2}{3}\right)^{\mu\left(t_{k} e_{n}, f\right)}
$$

for $w \in\left[t_{k} e_{n}, t_{k+1} e_{n}\right]$. Then $\rho\left(t_{k} e_{n}, t_{k+1} e_{n}\right)=\left|\log \frac{1}{2}\right|>0$ and by Lemma 5.3

$$
\left|f\left(e_{n}\right)\right| \leq 2^{A}\left(\frac{2}{3}\right)^{\mu\left(t_{k} e_{n}, f\right) A} ; A=\beta^{1+d_{6} \log \left(1 / t_{k}\right)}=\beta t_{k}^{\gamma}
$$

where $\gamma=d_{6} \log (1 / \beta)>0$. It follows from the hypothesis, that $f\left(e_{n}\right)=0$ and by similar argument, $f(x)=0$ for all $x \in\left(0, e_{n}\right]$. Hence $f \equiv 0$ and the claim (1) is proved.

(2) Let $g_{x, r}(z)$ be as above. By Theorem 3.4 we get

$$
g_{t_{k} e_{n}, t_{k}}(z) \leq d_{5}|z|^{\mu\left(t_{k} e_{n}, f\right)} \leq(1 / 3)^{\mu\left(t_{k} e_{n}, f\right)}
$$

for $z \in \mathbf{B}^{n}(0,1 / 4)$, where $\mu(x, f)$ is defined as above. Now

and it follows

$$
\frac{1}{2}\left|f(w)-f\left(t_{k} e_{n}\right)\right| \leq(1 / 3)^{\mu\left(t_{k} e_{n}, f\right)}
$$

$$
|f(w)| \leq 2(1 / 3)^{\mu\left(t_{k} e_{n}, f\right)}
$$

for $w \in E_{k}=\mathbf{B}^{n}\left(t_{k} e_{n}, t_{k} / 4\right)$. Let $E=\cup_{k \in \mathbb{N}} E_{k}$. It is clear, that $E$ is open and $\operatorname{rad} \underline{\operatorname{dens}}(E, 0)>0$. Then it follows by Lemma 2.22 that cap $\underline{\operatorname{dens}}(E, 0)>0$ and by Theorem 5.2 the mapping $f$ has the angular limit 0 at the origin if

$$
2(1 / 3)^{\mu\left(t_{k} e_{n}, f\right)} \rightarrow 0
$$

i.e. $\mu\left(t_{k} e_{n}, f\right) \rightarrow \infty$ as $k \rightarrow \infty$.

5.5. Lemma. Let $\alpha>1, k=1,2, \ldots$ Then there is $k_{0} \geq 1$ such that for $k \geq k_{0}$

$$
(\alpha-1) k / 2 \leq \rho\left(x_{k}, y_{k}\right) \leq 2(\alpha-1) k,
$$

where $x_{k}=e_{n} \sqrt{t_{k}^{2}+t_{k}^{2 \alpha}}, y_{k}=t_{k} e_{1}+t_{k}^{\alpha} e_{n}$ and $t_{k}=2^{-k}$.

Proof. Let $C=\sqrt{1+1 /(2 \alpha-2)}$. Then

$$
t_{k}^{2(1-\alpha)} \leq t_{k}^{2(1-\alpha)}+1 \leq C^{2} t_{k}^{2(1-\alpha)}
$$

and hence

$$
t_{k}^{1-\alpha} \leq \frac{\sqrt{t_{k}^{2}+t_{k}^{2 \alpha}}}{t_{k}^{\alpha}} \leq C t_{k}^{1-\alpha} .
$$

We note that

$$
\frac{1}{2} \exp (x) \leq \cosh (x) \leq \exp (x)
$$


for $x \geq 0$ and thus

$$
\frac{1}{2} \exp (\rho(x, y)) \leq 1+\frac{|x-y|^{2}}{2 x_{n} y_{n}} \leq \exp (\rho(x, y))
$$

for all $x, y \in \mathbf{H}^{n}$. Now

$$
\left|x_{k}-y_{k}\right|^{2}=2\left(t_{k}^{2}+t_{k}^{2 \alpha}-t_{k}^{\alpha} \sqrt{t_{k}^{2}+t_{k}^{2 \alpha}}\right)
$$

from which we obtain

$$
1+\frac{\left|x_{k}-y_{k}\right|^{2}}{2 x_{n} y_{n}}=\frac{\sqrt{t_{k}^{2}+t_{k}^{2 \alpha}}}{t_{k}^{\alpha}} .
$$

This, together with (5.6) and (5.7) gives us

$$
t_{k}^{1-\alpha} \leq \exp \left(\rho\left(x_{k}, y_{k}\right)\right) \leq 2 C t_{k}^{1-\alpha},
$$

or

$$
(1-\alpha) \log t_{k} \leq \rho\left(x_{k}, y_{k}\right) \leq(1-\alpha) \log t_{k}+\log 2 C .
$$

It follows that

$$
(\alpha-1) k / 2 \leq \rho\left(x_{k}, y_{k}\right) \leq 2(\alpha-1) k,
$$

for $k \geq \max \left\{1, \log (2 C)^{1 /(\alpha-1)}\right\}$.

5.8. Limits on a tangential set. The next result gives a criterion for a quasiregular mapping to have a limit along a tangential set. Note that the criterion of this result is in between the criteria (1) and (2) of Theorem 5.4.

5.9. Theorem. Let $f: \mathbf{H}^{n} \rightarrow \mathbf{B}^{n}$ be a $K$-quasiregular mapping with $f\left(2^{-k} e_{n}\right)=$ 0 , for all $k=1,2, \ldots$ and let $\gamma=d_{6} \log (1 / \beta), \mu_{k}=d_{3} i\left(2^{-k} e_{n}, f\right)^{1 /(n-1)}$, where $\beta \in(0,1), d_{6}>0$ are as in Lemma 5.3 and $d_{3} \in(0,1)$ is as in Lemma 3.4.

Fix $\alpha>1$. Suppose that

$$
\log \mu_{k}-2 \gamma(\alpha-1) k \rightarrow \infty \text { as } k \rightarrow \infty .
$$

Then $f$ has limit 0 at the origin along the tangential set $E=\left\{x=\left(x_{1}, 0, \ldots, 0, x_{n}\right): x_{n}=x_{1}^{\alpha}\right\}$.

Proof. As in the proof of Theorem 5.4, we obtain by Lemma 3.4 the estimate

$$
|f(z)| \leq(2 / 3)^{\mu_{k}} \equiv \varepsilon_{k}
$$

for $z \in\left[2^{-k-1} e_{n}, 2^{-k} e_{n}\right] \equiv F_{k}$. Let $x_{k}, y_{k}$ be as in Lemma 5.5 and $\beta \in(0,1)$, $d_{6}>0$ be the constants of Lemma 5.3 corresponding to $F_{k}$ and depending only on $n, K$. We may conclude by Lemma 5.3 that

$$
\left|f\left(t e_{1}+t^{\alpha} e_{n}\right)\right|<\varepsilon_{k}^{A} ; \quad A=\beta^{1+d_{6} \rho\left(x_{k+1}, y_{k+1}\right)}
$$

for $t \in\left[2^{-k-1}, 2^{-k}\right]$ as $\rho\left(t e_{1}+t^{\alpha} e_{n}, F_{k}\right) \leq \rho\left(x_{k+1}, y_{k+1}\right)$.

It is sufficient to show that

$$
\varepsilon_{k}^{A} \rightarrow 0 \text { as } k \rightarrow \infty
$$


By Lemma 5.5 this follows if

$$
\beta^{1+2 d_{6}(\alpha-1) k} \log \frac{1}{\varepsilon_{k}} \rightarrow \infty \text { as } k \rightarrow \infty,
$$

or

$$
2 d_{6} k(\alpha-1) \log \beta+\log \log \frac{1}{\varepsilon_{k}} \rightarrow \infty .
$$

This can be written

$$
-2 \gamma(\alpha-1) k+\log \mu_{k} \rightarrow \infty
$$

5.10. Conical uniform continuity. Let $f$ be a quasiregular mapping from the upper half space into a ball. We study $f$ on a cone contained in the upper half space fixed at the origin. We assume that the function has a limit at the origin along a fixed sequence of points and derive an explicit upper bound for the speed of convergence in the cone.

5.11. Theorem. Let $f: \mathbf{H}^{n} \rightarrow \mathbf{B}^{n}$ be $K$-quasiregular, $f\left(2^{-j} e_{n}\right) \rightarrow \alpha$, with $i\left(2^{-j} e_{n}, f\right)^{1 /(n-1)}=j$, and let $x \in C(\varphi)$ for some $\varphi \in(0, \pi / 2)$. Let $k$ be such that $2^{-k-1}<|x| \leq 2^{-k}$. Then

$$
|f(x)-\alpha| \leq M(|x|, n, K, \varphi) \rightarrow 0 \text { as }|x| \rightarrow 0,
$$

where

$$
M(|x|, n, K, \varphi)=2\left(\frac{2-s}{1-s}\right) s^{k} \leq \frac{4}{s(1-s)}|x|^{-\log _{2} s},
$$

$R_{\varphi}>0$ is a constant depending only on $\varphi, s=(2 / 3)^{d_{3} A} \in(0,1), A=\beta^{1+d_{6} R_{\varphi}} \in$ $(0,1)$ depend only on $n, K, \varphi$ and $\beta \in(0,1), d_{3} \in(0,1), d_{6}>1$ are constants depending only on $n, K$ as in Lemma 4.3. In particular, $-\log _{2} s=d_{3} A \log _{2}(3 / 2) \in$ $(0,1)$.

Proof. Fix $x \in C(\varphi)$ and let $\alpha_{k}=f\left(2^{-k} e_{n}\right)$. There exists a constant $R_{\varphi}$ depending only on $\varphi$ such that $x \in D\left(2^{-k} e_{n}, R_{\varphi}\right)$. By [Vuo88, 4.23], we have the following upper bound for $R_{\varphi}$ :

$$
R_{\varphi} \leq \log \left(\frac{9(1+u)^{2}}{8 \cos ^{2} \varphi}\right)
$$

where $u^{2}=\sin ^{2} \varphi+\left(\cos ^{2} \varphi\right) / 9$.

Let $g_{k}$ be a Möbius transformation such that $g_{k}(0)=2^{-k} e_{n}$ and $g_{k} \mathbf{B}^{n}=\mathbf{H}^{n}$. Then the mapping $h_{k}(x)$ defined by

$$
h_{k}(x)=\left(f \circ g_{k}(x)-\alpha_{k}\right) / 2
$$

is $K$-quasiregular on $\mathbf{B}^{n}, h_{k}: \mathbf{B}^{n} \rightarrow \mathbf{B}^{n}$ and $h_{k}(0)=0$. We apply Lemma 4.3 with $r=1 / 2$ to obtain

$$
\left|h_{k}(y)\right| \leq(2 / 3)^{d_{3} k A} \text { for } y \in D_{\mathbf{B}^{n}}\left(0, R_{\varphi}\right),
$$


where $A=\beta^{1+d_{6}} R_{\varphi} \in(0,1)$ and $\beta, d_{6}$ are as in Lemma 4.3. Hence

$$
\left|f(x)-\alpha_{k}\right| \leq 2(2 / 3)^{d_{3} k A}
$$

and by the triangle inequality

$$
|f(x)-\alpha| \leq 2(2 / 3)^{d_{3} k A}+M_{k},
$$

where $M_{k}=\left|\alpha_{k}-\alpha\right|$. It remains to estimate $M_{k}$.

As $2^{-k-1} e_{n} \in D\left(2^{-k} e_{n}, R_{\varphi}\right)$, by (5.12), we have the upper bound

$$
\frac{1}{2}\left|\alpha_{k+1}-\alpha_{k}\right|=\left|h_{k}\left(y_{k}\right)\right| \leq(2 / 3)^{d_{3} k A}
$$

where $y_{k}=g_{k}^{-1}\left(2^{-k-1} e_{n}\right)$. Hence

$$
\left|\alpha_{k}-\alpha\right| \leq \sum_{j=k}^{\infty}\left|\alpha_{j+1}-\alpha_{j}\right| \leq 2 \sum_{j=k}^{\infty}(2 / 3)^{d_{3} j A}
$$

Let $s=(2 / 3)^{d_{3} A}$. Then

$$
\sum_{j=k}^{\infty}(2 / 3)^{d_{3} j A}=\frac{s}{1-s}-\frac{s\left(1-s^{k-1}\right)}{1-s}=\frac{s^{k}}{1-s} .
$$

This together with (5.13) gives

$$
|f(x)-\alpha| \leq 2\left(\frac{2-s}{1-s}\right) s^{k} .
$$

As $k+1 \geq-\log _{2}|x|$, we have

$$
s^{k+1} \leq s^{-\log _{2}|x|}=|x|^{-\log _{2} s} .
$$

This together with (5.14) gives the second inequality.

5.15. Theorem. Let $f: \mathbf{H}^{n} \rightarrow \mathbf{B}^{n}$ be $K$-quasiregular, $\varphi \in(0, \pi / 2)$, and let $\left(b_{k}\right), f\left(b_{k}\right)=0$ be a sequence of points in $\mathbf{H}^{n}$ with $b_{k} \rightarrow 0$ as $k \rightarrow \infty$. If

$$
i\left(b_{k}, f\right)\left(b_{k}\right)_{n}^{d_{10}} \rightarrow \infty
$$

as $k \rightarrow \infty$, then $f \equiv 0$. Here $d_{10}>0$ is a constant depending only on $n, K$.

In particular, if the points $\left(b_{k}\right)$ are in $C(\varphi)$ and

$$
i\left(b_{k}, f\right)\left|b_{k}\right|^{d_{10}} \rightarrow \infty
$$

as $k \rightarrow \infty$, then $f \equiv 0$.

Proof. Let $g_{k}$ be a Möbius transformation such that $g_{k}\left(\mathbf{B}^{n}\right)=\mathbf{H}^{n}$ and $g_{k}(0)=$ $b_{k}$. We let $h_{k}=f \circ g_{k}$ and $r=1 / 2$. Now $h_{k}: \mathbf{B}^{n} \rightarrow \mathbf{B}^{n}$ is a $K$-quasiregular mapping with $h_{k}(0)=0$. By applying Lemma 4.3, we obtain the inequality

$$
\left|h_{k}(y)\right| \leq(2 / 3)^{\mu_{k} A_{k}} ; \quad y \in D\left(b_{k}, M_{k}\right),
$$

for $M_{k}>0$, where $A_{k}=\beta^{1+d_{6} M_{k}}$ and $\mu_{k}=d_{3} i\left(0, h_{k}\right)^{1 /(n-1)}$. 
Next we find $M_{k}$ such that $e_{n} \in D\left(b_{k}, M_{k}\right)$. We may assume that $\left|b_{k}\right| \leq 1$. Then by $(2.2)$

$$
\cosh \rho\left(e_{n}, b_{k}\right)=1+\frac{\left|b_{k}-e_{n}\right|^{2}}{2\left(b_{k}\right)_{n}} \leq 1+\frac{2}{\left(b_{k}\right)_{n}} .
$$

Since for $t \geq 2$,

$$
\log \left(1+t+\sqrt{(t+1)^{2}-1}\right) \leq \log (3 t)
$$

it follows that

$$
\rho\left(e_{n}, b_{k}\right) \leq \log \left(6 /\left(b_{k}\right)_{n}\right)
$$

for $\left(b_{k}\right)_{n} \leq 1$. We choose $M_{k}=\log \left(6 /\left|b_{k}\right|\right)$. This together with (5.16) yields

$$
\left|f\left(e_{n}\right)\right| \leq(2 / 3)^{\mu_{k} B_{k}} ; \quad B_{k}=\beta^{1+d_{6} \log \left(6 /\left(b_{k}\right)_{n}\right)}
$$

for $k$ large enough. To obtain the result, we want that

$$
(2 / 3)^{\mu_{k} B_{k}} \rightarrow 0
$$

or equivalently

$$
\mu_{k} B_{k} \log (2 / 3) \rightarrow-\infty
$$

This can be written as

$$
\mu_{k} \beta^{1+d_{6} \log \left(1 /\left(b_{k}\right)_{n}\right)+d_{6} \log 6} \rightarrow \infty,
$$

which, in turn, is equivalent to

$$
i\left(b_{k}, f\right)^{1 /(n-1)}\left(b_{k}\right)_{n}^{d_{6} \log (1 / \beta)} \rightarrow \infty .
$$

By a similar argument, $f(z)=0$ for every $z \in\left(0, e_{n}\right]$, and hence $f \equiv 0$. By choosing $d_{10}=d_{6} \log (1 / \beta)(n-1)$ the claim follows.

We note that if $\left(b_{k}\right)$ is a sequence of points in $C(\varphi)$, then

$$
\left|b_{k}\right| /\left(b_{k}\right)_{n} \leq 1 / \sin (\pi / 2-\varphi)
$$

and hence by (5.17),

$$
\left|f\left(e_{n}\right)\right| \leq(2 / 3)^{\mu_{k} \tilde{B}_{k}} ; \quad \tilde{B}_{k}=\beta^{1+d_{6} \log \left(1 /\left|b_{k}\right|\right)-d_{6} \log (\sin (\pi / 2-\varphi) / 6)},
$$

which yields the final observation.

5.19. Interpolation sequences. A sequence $\left\{z_{k}\right\}$ of points in the unit disk $\mathbf{B}^{2}$ is called a Blaschke sequence if

$$
\sum_{k=1}^{\infty}\left(1-\left|z_{k}\right|\right)<\infty
$$

It is called uniformly separated if

$$
\prod_{j \neq k}\left|\frac{z_{j}-z_{k}}{1-\bar{z}_{j} z_{k}}\right| \geq \delta, \quad k=1,2, \ldots
$$

for some constant $\delta>0$ that is independent of $k$. 
A sequence $\left\{z_{k}\right\}$ is said to be an interpolation sequence if for each complex sequence $\left\{w_{k}\right\} \in \ell^{\infty}$ there exists a bounded analytic function $f$ with $f\left(z_{k}\right)=w_{k}$ for $k=1,2, \ldots$. Carleson proved in 1958 that $\left\{z_{k}\right\}$ is an interpolation sequence if and only if it is uniformly separated.

5.20. Lemma. The sequence $z_{k}=1-e^{-k}$ is uniformly separated.

Proof. Let $k \geq 1$ and let

$$
A_{k}=\prod_{j \neq k}\left|\frac{e^{-k}-e^{-j}}{e^{-k}+e^{-j}-e^{-(j+k)}}\right| .
$$

Then

$$
A_{k} \geq \prod_{j \neq k}\left|\frac{e^{-k}-e^{-j}}{e^{-k}+e^{-j}}\right|=\prod_{j \neq k}\left|\frac{1-e^{-j+k}}{1+e^{-j+k}}\right|
$$

We note that

$$
\frac{e^{n}-1}{e^{n}+1}=\frac{1-e^{-n}}{e^{-n}+1}
$$

and hence

$$
A_{k} \geq\left(\prod_{n=1}^{\infty}\left|\frac{1-e^{-n}}{1+e^{-n}}\right|\right)^{2}
$$

Because

$$
\frac{1-e^{-n}}{1+e^{-n}} \geq 1-e^{-n+\log 2},
$$

we conclude that there is $\delta_{0} \in(0,1)$ such that

$$
\prod_{n=1}^{\infty}\left|\frac{1-e^{-n}}{1+e^{-n}}\right| \geq \delta_{0} \prod_{n=1}^{\infty}\left(1-e^{-n}\right) .
$$

Clearly

$$
\prod_{n=1}^{\infty}\left(1-x^{-n}\right)=\delta_{1}>0
$$

for $x>1$. By choosing $\delta=\left(\delta_{0} \delta_{1}\right)^{2}$ the claim follows.

5.21. Example. (by P. Lappan [Lap03]) We construct a Blaschke product $f: \mathbf{B}^{2} \rightarrow \mathbf{B}^{2}$ with $f\left(\tilde{b}_{k}\right)=0$ for $\tilde{b}_{k} \in(0,1)$ with $\tilde{b}_{k}<\tilde{b}_{k+1}$ such that there is a set $E$ with $\left\{\tilde{b}_{k}\right\} \in E, \lim _{x \rightarrow 1, x \in E} f(x)=0$, and $\theta^{* 2}(E, 1)=\theta^{2}\left(\mathbf{B}^{2}, 1\right)$ but $f$ fails to have an angular limit at 1.

Let $b_{k}=1-e^{-k}$. By Lemma 5.20 this sequence is uniformly separated and hence an interpolation sequence. If we form the Blaschke product $B(z)$ having zeros (of order one) at the points $b_{k}$, then $B(z)$ does not have a radial limit at $z=1$, since there is a sequence $\left\{c_{k}\right\} \subset(0,1)$ such that $b_{k-1}<c_{k}<b_{k}$ and a number $\sigma>0$ such that $\left|B\left(c_{k}\right)\right| \geq \sigma$ for each $k$. This holds by (14) in [BS60, Example 4]. 
Let $\left\{m_{k}\right\}$ be any sequence of positive integers with $m_{k} \rightarrow \infty$ as $k \rightarrow \infty$, and let $n_{k}=m_{k}+2 \sum_{j=1}^{k-1} m_{j}$. Note that $n_{k+1}-n_{k}=m_{k}+m_{k+1}$. Let $a_{k}=b_{n_{k}}$, let $p_{k}=n_{k}+m_{k}$, and let $d_{k}=c_{p_{k}}$. We now have that

$$
a_{k}=b_{n_{k}}<b_{n_{k}+m_{k}-1}<c_{p_{k}}=d_{k}<b_{p_{k}}<b_{p_{k}+m_{k+1}-1}<a_{k+1} .
$$

Now define

$$
B_{0}(z)=\prod_{k=1}^{\infty}\left(\frac{\left|a_{k}\right|\left(a_{k}-z\right)}{a_{k}\left(1-\bar{a}_{k} z\right)}\right)^{m_{k}}
$$

Next we show that $\left|B_{0}\left(d_{k}\right)\right| \geq\left|B\left(d_{k}\right)\right|$.

Just comparing the position of factors, we have that, for $q<k, a_{q}=b_{n_{q}}<$ $b_{n_{q}+1}$ and hence

$$
\left(\frac{\left|d_{k}-a_{q}\right|}{\left|1-\bar{a}_{q} d_{k}\right|}\right)^{m_{q}}>\prod_{j=n_{q}+1}^{n_{q}+m_{q}} \frac{\left|d_{k}-b_{j}\right|}{\left|1-\bar{b}_{j} d_{k}\right|} .
$$

Also, for $q \geq k$, as $n_{q+1}-m_{q+1}=n_{q}+m_{q}$, we have

$$
\left(\frac{\left|d_{k}-a_{q}\right|}{\left|1-\bar{a}_{q} d_{k}\right|}\right)^{m_{q}}>\prod_{j=n_{q}-m_{q}+1}^{n_{q}} \frac{\left|d_{k}-b_{j}\right|}{\left|1-\bar{b}_{j} d_{k}\right|} .
$$

Essentially, using the points $c_{p_{k}}=d_{k}$, we have that the corresponding zeros of $B_{0}(z)$, counted according to multiplicity, are farther away from the points $d_{k}$ than the corresponding zeros of $B(z)$. All of the factors of $B_{0}$ are considered, but there are some factors of $B(z)$ not counted in this scheme. Now $i\left(a_{k}, B_{0}\right) \rightarrow \infty$ as $k \rightarrow \infty$ and by Lemma 4.8 the claim follows.

5.22. Remark. The conditions of Theorem 5.15 are not satisfied in the setting of Example 5.21. More precisely, we show by comparing $m_{k}=i\left(a_{k}, B_{0}\right)$ to $1-\left|a_{k}\right|$ that a condition of the type

$$
i\left(a_{k}, B_{0}\right)\left(1-\left|a_{k}\right|\right)^{\alpha} \rightarrow \infty
$$

does not hold for $\alpha=d_{10}>0$ as in Theorem 5.15 (or for any $\alpha>0$ ).

To see this, consider the zeroes of the mapping $f$ at the points

$$
a_{k}=1-\exp \left(-m_{k}-2 \sum_{j=1}^{k-1} m_{j}\right) .
$$

Let $n_{k}=m_{k}+2 \sum_{j=1}^{k-1} m_{j}$. By (2.3) we have the formula

$$
\sinh ^{2}\left(\frac{1}{2} \rho\left(a_{k}, 0\right)\right)=\left(1-e^{-n_{k}}\right) e^{n_{k}}=e^{n_{k}}-1 .
$$

This gives us

$$
\rho\left(a_{k}, 0\right)=2 \log \left(e^{n_{k}}-1+2 \sqrt{e^{2 n_{k}}-e^{n_{k}}}+e^{n_{k}}\right) .
$$

From (5.23) we obtain the following bounds for $\rho\left(a_{k}, 0\right)$ :

$$
n_{k} \leq \rho\left(a_{k}, 0\right) \leq n_{k}+\log 4 \text {. }
$$


Let $h$ be a Möbius transformation such that $h\left(\mathbf{B}^{2}\right)=\mathbf{H}^{2}$ and $h\left(\left[0, e_{1}\right)\right)=$ $\left[e_{2}, 0\right)$, and let $b_{k}=h\left(a_{k}\right)$. Now $g=f \circ h^{-1}$ is a quasiregular mapping, $g: \mathbf{H}^{2} \rightarrow$ $\mathbf{B}^{2}$, as in Theorem 5.15. Then by $(2.2)$

$$
\cosh \left(\rho\left(b_{k}, e_{2}\right)\right)=1+\frac{\left(1-\left|b_{k}\right|\right)^{2}}{2\left|b_{k}\right|}=\frac{\left|b_{k}\right|-1}{2}+\frac{1}{2\left|b_{k}\right|} .
$$

From (5.25) we see that

$$
\frac{1}{2} \exp \left(\rho\left(b_{k}, e_{2}\right)\right) \leq \cosh \left(\rho\left(b_{k}, e_{2}\right)\right) \leq \frac{1}{2\left|b_{k}\right|},
$$

and

$$
\frac{1}{2} \exp \left(\rho\left(b_{k}, e_{2}\right)\right)+\frac{1}{2} \geq \cosh \left(\rho\left(b_{k}, e_{2}\right)\right) \geq \frac{1}{2\left|b_{k}\right|}-\frac{1}{2} .
$$

From (5.26) and (5.27) we obtain the inequalities

$$
\frac{1}{3} \exp \left(-\rho\left(b_{k}, e_{2}\right)\right) \leq\left|b_{k}\right| \leq \exp \left(-\rho\left(b_{k}, e_{2}\right)\right) .
$$

Combining (5.28) and (5.24) yields

$$
\frac{1}{12} \exp \left(-n_{k}\right) \leq\left|b_{k}\right| \leq \exp \left(-n_{k}\right)
$$

Next we estimate the quantity

$$
i\left(b_{k}, f\right)^{1 /(n-1)}\left|b_{k}\right|^{d_{6} \log (1 / \beta)}=m_{k} \exp \left(-n_{k} d_{6} \log (1 / \beta)\right)
$$

from Theorem 5.15. As $m_{k} \leq n_{k}$, we have

$$
m_{k} \exp \left(-n_{k} d_{6} \log (1 / \beta)\right) \leq m_{k} \exp \left(-m_{k} d_{6} \log (1 / \beta)\right) \rightarrow 0,
$$

as $k \rightarrow \infty$, proving the claim.

5.30. Quasiconformal mappings. We give a bound for how close to a point $\alpha$ the values attained by a quasiconformal mapping on a sequence of continua approaching the boundary can be. The bound is given in terms of the diameter of the continua involved. In order to prove this result, we need the following lemmas.

5.31. Lemma. Let $w>0$ and $t \in\left(0, \min \left\{w^{2}, 1 / w\right\}\right)$. Then

$$
\frac{1}{2} \log \frac{1}{t}<\log \frac{w}{t}<2 \log \frac{1}{t} .
$$

Proof. For $w=1$ the claim is trivial. We study the cases $w>1$ and $w \in(0,1)$ separately. 
Let $w>1$. Then $1 / w>t$. Clearly

$$
\begin{aligned}
\log \frac{1}{t} & <\log \frac{w}{t} \\
& =\left(\log \frac{1}{t}\right)\left(1+\frac{\log w}{\log (1 / t)}\right) \\
& <\left(\log \frac{1}{t}\right)\left(1+\frac{\log w}{\log w}\right)=2 \log \frac{1}{t} .
\end{aligned}
$$

Let $w \in(0,1)$. Then

$$
\log \frac{1}{t}>\log \frac{w}{t}=\left(\log \frac{1}{t}\right)\left(1-\frac{\log (1 / w)}{\log (1 / t)}\right) .
$$

Now $t<w^{2}$ and hence $\log \left(1 / w^{2}\right)<\log (1 / t)$. It follows that

$$
\frac{\log (1 / w)}{\log (1 / t)}<\frac{1}{2}
$$

and by (5.32) we obtain $\log \frac{w}{t}>\frac{1}{2} \log \frac{1}{t}$.

5.33. Lemma. Let $C \subset \mathbf{B}^{n}$ be connected and $0<d(C) \leq 1$. Then $m \equiv$ $d(0, C) / d(C)<\infty$ and if $m>0$, then

$$
\mathrm{M}(\Gamma) \geq \frac{1}{2} \tau\left(4 m^{2}+4 m\right) \geq 2^{-n} \tau(m) ; \quad \Gamma=\Delta\left(\overline{\mathbf{B}}^{n}(1 / 2), C ; \mathbf{B}^{n}\right) .
$$

Proof. The second inequality holds by $[\mathrm{Vuo} 88,5.63$.$] . To prove the first inequal-$ ity, we note that if $C \cap \overline{\mathbf{B}}^{n}(1 / 2) \neq \emptyset$, then $\mathrm{M}(\Gamma)=\infty$ and there is nothing to prove. In what follows we may assume that $C \cap \overline{\mathbf{B}}^{n}(1 / 2)=\emptyset$. Now the result follows from the symmetry property of the modulus [Vuo88, Lemma 5.22.] and the estimate [Vuo88, Lemma 7.38.].

5.34. Theorem. Let $f: \mathbf{B}^{n} \rightarrow \mathbb{R}^{n}$ be a quasiconformal mapping or constant, $\alpha \in \mathbb{R}^{n}$ and $\left(C_{j}\right)$ a sequence of nondegenerate continua such that $C_{j} \rightarrow \partial \mathbf{B}^{n}$ and $|f(x)-\alpha|<M_{j}$ when $x \in C_{j}$, where $M_{j} \rightarrow 0$ as $j \rightarrow \infty$. If

$$
\limsup _{j \rightarrow \infty} \tau\left(\frac{1}{d\left(C_{j}\right)}\right)\left(\log \frac{1}{M_{j}}\right)^{n-1}=\infty,
$$

then $f \equiv \alpha$. In particular, if

$$
\limsup _{j \rightarrow \infty}\left(\log \frac{1}{d\left(C_{j}\right)}\right)^{1-n}\left(\log \frac{1}{M_{j}}\right)^{n-1}=\infty
$$

then $f \equiv \alpha$.

Proof. Suppose that $f$ is not constant. Let $\Gamma_{j}=\Delta\left(\mathbf{B}^{n}(1 / 2), C_{j} ; \mathbb{R}^{n}\right)$. Then by Lemma 5.33

$$
\mathrm{M}\left(\Gamma_{j}\right) \geq 2^{-n} \tau\left(\frac{d\left(0, C_{j}\right)}{d\left(C_{j}\right)}\right) \geq 2^{-n} \tau\left(\frac{1}{d\left(C_{j}\right)}\right) .
$$


Let $w=d\left(f \overline{\mathbf{B}}^{n}(1 / 2), \alpha\right)>0$. Now by Lemma 2.10

$$
\mathrm{M}\left(f \Gamma_{j}\right) \leq \omega_{n-1}\left(\log \frac{w}{M_{j}}\right)^{1-n} \leq \omega_{n-1}\left(\frac{1}{2} \log \frac{1}{M_{j}}\right)^{1-n},
$$

whenever $M_{j}<\min \left\{w^{2}, 1 / w\right\}$ by Lemma 5.31. Because $\mathrm{M}\left(\Gamma_{j}\right) \leq K\left(\mathrm{M}\left(f \Gamma_{j}\right)\right)$, the estimates above yield

$$
\tau\left(\frac{1}{d\left(C_{j}\right)}\right)\left(\log \frac{1}{M_{j}}\right)^{n-1} \leq 2^{2 n-1} K \omega_{n-1},
$$

proving the first part of the claim.

The estimate (2.12) yields

$$
\tau(t) \geq 2^{1-n} \omega_{n-1}\left[\log \left(\frac{\lambda_{n}}{2}(\sqrt{1+t}+\sqrt{t})\right)\right]^{1-n},
$$

where $t=1 / d\left(C_{j}\right)$. It follows that

$$
\begin{aligned}
{\left[\log \left(\frac{\lambda_{n}}{2}(\sqrt{1+t}+\sqrt{t})\right)\right]^{1-n} } & \geq\left[\log \left(\frac{\lambda_{n}}{2}(1+2 \sqrt{t})\right)\right]^{1-n} \\
& =\left[\log \left(\frac{\lambda_{n}}{2}\left(1+\frac{2}{\sqrt{d\left(C_{j}\right)}}\right)\right)\right]^{1-n} .
\end{aligned}
$$

We note that

$$
\left[\log \left(\frac{\lambda_{n}}{2}\left(1+\frac{2}{\sqrt{d\left(C_{j}\right)}}\right)\right)\right]^{1-n} \geq\left[2 \log \left(\frac{\lambda_{n}}{\sqrt{d\left(C_{j}\right)}}\right)\right]^{1-n}
$$

whenever $j$ is large enough. Let $v=\lambda_{n}$. Now by Lemma 5.31

$$
\left[2 \log \left(\frac{\lambda_{n}}{\sqrt{d\left(C_{j}\right)}}\right)\right]^{1-n} \geq\left(2 \log \frac{1}{d\left(C_{j}\right)}\right)^{1-n}
$$

for $\sqrt{d\left(C_{j}\right)}<\min \left\{v^{2}, 1 / v\right\}$. Hence

$$
\tau\left(\frac{1}{d\left(C_{j}\right)}\right)\left(\log \frac{1}{M_{j}}\right)^{n-1} \geq 2^{2-2 n} \omega_{n-1}\left(\log \frac{1}{d\left(C_{j}\right)}\right)^{1-n}\left(\log \frac{1}{M_{j}}\right)^{n-1}
$$

which gives the second part of the claim.

Theorem 5.34 is an improvement of the following result from [Vuo85]:

5.35. Theorem. [Vuo85, Corollary 4.9] Let $f: \mathbf{B}^{n} \rightarrow \mathbb{R}^{n}$ be a quasiconformal mapping or a constant, let $\alpha \in \mathbb{R}^{n}$, and let $\left(C_{j}\right)$ be a sequence of connected sets such that $C_{j} \rightarrow \partial \mathbf{B}^{n}$ and $|f(x)-\alpha|<M_{j}$ for $x \in C_{j}$ where $M_{j} \rightarrow 0$ as $j \rightarrow \infty$. If

$$
\limsup _{j \rightarrow \infty} d\left(C_{j}\right)\left(\log \frac{1}{M_{j}}\right)^{n-1}=\infty
$$

then $f \equiv \alpha$. 


\section{REFERENCES}

[AVV97] G. D. Anderson, M. K. Vamanamurty and M. Vuorinen: Conformal invariants, inequalities and quasiconformal mappings, Wiley-Interscience, 1997.

[BS60] F. Bageminl And W. Seidel: Sequential and continuous limits of meromorphic functions, Ann. Acad. Sci. Fenn. Ser. A I 280 (1960) 1-17.

[Bie31] L. Bieberbach: Lehrbuch der Funktionentheorie, Bd. 2, Moderne Funktionentheorie, Leipzig, Teubner, 1931.

[Car50] C. CARAThÉOdORY: Funktionentheorie, Bd. 1, Basel, Birkhäuser, 1950.

[EE02] V. Eiderman And M. EssÉn: Uniqueness Theorems of Analytic and Subharmonic Functions, Algebra i Analiz 14, no. 6 (2002), 1-88.

[Geh63] F. W. GEHRING: The Carathéodory convergence theorem for quasiconformal mappings in the space, Ann. Acad. Sci. Fenn. Ser. A I 336/11 (1963), 1-21.

[Jär00] P. JÄRVI: On the zeros and growth of quasiregular mappings, J. Anal. Math. 82 (2000), 347-362.

[Lap03] P. LAPPAN: Private communication February 2003, (email to Matti Vuorinen), 2003.

[Res89] Yu. G. Reshetnyak: Space Mappings with Bounded Distortion, Translations of Mathematical Monographs 73, Amer. Math. Soc., Providence, 1989.

[Ric80a] S. Rickman: On the number of omitted values of entire quasiregular mappings, J. Anal. Math. 37 (1980), 100-117.

[Ric80b] S. Rickman: Asymptotic values and angular limits of quasiregular mappings of a ball, Ann. Acad. Sci. Fenn. Ser. A I Math. 5 (1980), 185-196.

[Ric93] S. Rickman: Quasiregular Mappings, Ergeb. Math. Grenzgeb. (3), Vol. 26, Springer-Verlag, Berlin, 1993.

[RS86] S. Rickman and U. SRebro: Remarks on the local index of quasiregular mappings, J. Anal. Math. 46 (1986), 246-250.

[Run68] D. C. RunG: Behavior of holomorphic functions in the unit disk on arcs of positive diameter, J. Math. Kyoto Univ. 8-3 (1968), 417-464.

[Väi71] J. VÄIsÄLÄ: Lectures on n-Dimensional Quasiconformal Mappings, Lecture Notes in Math., Vol. 229, Springer-Verlag, Berlin, 1971.

[Vuo80] M. VuORINEn: On the existence of angular limits of n-dimensional quasiconformal mappings, Ark. Mat. 18 (1980), 157-180.

[Vuo81] M. VuORINEn: Capacity densities and angular limits of quasiregular mappings, Trans. Amer. Math. Soc. 263 (1981), 343-354.

[Vuo85] M. Vuorinen: Koebe Arcs and Quasiregular Mappings, Math. Z. 190 (1985), 95106.

[Vuo88] M. Vuorinen: Conformal Geometry and Quasiregular Mappings, Lecture Notes in Math., Vol. 1319, Springer-Verlag, Berlin, 1988. 